CPTH-S590.1297

hep-th/9712153

\title{
Implementation of an iterative map in the construction of (quasi)periodic instantons: chaotic aspects and discontinuous rotation numbers.
}

\author{
A. Chakrabarti币 \\ ECentre de Physique Théorique, Ecole Polytechnique \\ 91128 Palaiseau, France.
}

\begin{abstract}
An iterative map of the unit disc in the complex plane (Appendix) is used to explore certain aspects of selfdual, four dimensional gauge fields (quasi)periodic in the Euclidean time. These fields are characterized by two topological numbers and contain standard instantons and monopoles as different limits. The iterations do not correspond directly to a discretized time evolution of the gauge fields. They are implemented in an indirect fashion. First, $(t, r, \theta, \phi)$ being the standard coordinates, the $(r, t)$ half plane is mapped on the unit disc in an appropriate way. This provides an $(r, t)$ parametrization (Sec.1) of $Z_{0}$, the starting point of the iterations and makes the iterates increasingly complex functions of $r$ and $t$. These are then incorporated as building blocks in the generating function of the fields (Sec.2). We explain (starting in Sec.1 and at different stages) in what sense and to what extent some remarkable features of our map (indicated in the title) are thus carried over into the continuous time development of the fields. Special features for quasiperiodicity are studied (Sec.3). Spinor solutions (Sec.4) and propagators (Sec.5) are discussed from the point of view of the mapping. Several possible generalizations are indicated (Sec.6). Some broader topics are discussd in conclusion (Sec.7).
\end{abstract}

\footnotetext{
${ }^{1}$ chakra@pth.polytechnique.fr

${ }^{2}$ Laboratoire Propre du CNRS UPR A.0014
} 


\section{Introduction}

An iterative map of the unit disc centered at the origin of the complex plane is studied in the Appendix(App). The motivation is that it can be implemented fruitfully in the study of a hierarchy of fourdimensional, selfdual, (quasi) periodic gauge fields. Fields with spherical symmetry in $R^{3}$ are mostly used to illustrate our approach. More general possibilities are indicated at the end(Sec.6).Such fields have been studied previously in a series of papers[1,2, 3, 4, 5, 6, 7, 8] which contains references to other sources. Here we reformulate them from the point of view of the iterative map. This brings remarkable new aspects to light.

Our gauge fields are are (quasi) periodic in Euclidean time. The basic ingredient ( one may say the generating function ) for the spherically symmetric fields is a holomorphic function $g(r+i t)$ satisfying certain constraints (Sec.2) in the $(r, t)$ half plane $(r \geq 0)$. Here $(t, r, \theta, \phi)$ are the standard coordinates.The function $g$ can have several factors ( or nonfactorized terms) each with its own period in $t$. When all the periods are mutually commensurable the lowest common multiple of the component periods is the overall one. This is the periodic case. When at least one of the component periods is incommensurable with some others, one has, by definition quasiperiodicity.

As is well-known, fields periodic in theEuclidean time provide one possible approach to field theory at finite temperature. Our explicit constructions are such that even an infinitesimal change in one single parameter (determining one of the component periods) can make a periodic solution quasiperiodic and vice versa. So they are considered in a parallel fashion.

The map studied in App is

$$
Z_{p+1}=\frac{a_{p}+Z_{p}}{\bar{a}_{p}+Z_{p}^{-1}}, \quad\left|Z_{0}\right| \leq 1 ; 0<\left|a_{p}\right|<1
$$

Note the inverse $\left(Z_{p}{ }^{-1}\right)$ in the denominator. Possible choices of $a_{p}$ are discussed in App. For implementing the map in the construction of the gauge fields the crucial step is a suitable $(r, t)$ parametrization of $Z_{0}$. The two choices considered are

$$
Z_{0}=e^{-k(r+i t)} \quad(k>0)
$$


and

$$
Z_{0}=\frac{\sum_{l=1}^{n} \lambda_{l}^{2}\left(e^{k_{l}\left(r+i t-i c_{l}\right)}-1\right)^{-1}}{\sum_{l=1}^{n} \lambda_{l}^{2}\left(1-e^{-k_{l}\left(r+i t-i c_{l}\right)}\right)^{-1}}
$$

with real parameters $(\lambda, k, c)$ and

$$
k_{n}>k_{(n-1)}>\ldots \ldots>k_{2}>k_{1}>0 .
$$

The uses of (2) will be amply illustrated (Secs.2,3 and App.). The choice (3) is particularly suited to the construction of spinor solutions (Sec.4). It can be shown to satisfy all the necessary constraints.

With $Z_{0}$ thus chosen one can set (Sec.2) the generating function of the gauge fields to be

$$
g=\left(\prod_{j=1}^{n} Z_{p_{j}}^{(j)}\right)
$$

where different sets of parameters are associated to each factor.

The iterations do not correspond to a discretized time evolution of the fields. At each step one has a different action ( Sec.2), a different system. But the $(r, t)$ parametrization of $Z_{0}^{(j)}$ will provide the key to the usefulness of the iterations. As $p_{j}$ increases $g$ becomes a more and more complicated function of $(r, t)$. But the fact that such complications are introduced in a very specific fashion stepwise makes some remarkable properties readily accessible. Some crucial properties of each block $Z_{p_{j}}^{(j)}$ as a whole are delivered directly. To give this statement explicit content let us look at the two most striking features of our map.( Upto Sec.4, (2) will usually be referred to directly for simplicity. This is not an essential restriction.)

(1). For $r=0$ (the circumference of the unit disc) $Z_{p}$ for any $\mathrm{p}$, is a phase, the angle being denoted by $\psi_{p}$, and (1) becomes a circle map. This map (App) satisfies all the criteria for being chaotic ( [9], Def.8.5, p.50). These are the following ones.

(a) A sensitive dependence on initial data, encoded by a positive characteristic index.

(b) A dense set of periodic points.

(c) Transitivity.

Moreover this phase and its derivatives provide the coefficients of a series expansion in $r$ of $Z$ or $g$ (App). 
Suppose now, that as a consequence of (a), namely the positive index, at a certain level $l$ of iterations the difference

$$
\psi_{l}\left(\psi_{0}+\delta \psi_{0}\right)-\psi_{l}\left(\psi_{0}\right)
$$

is appreciably large even for a small $\delta \psi_{0}$ (considering $\psi_{l}$ as a function of its initial datum $\left.\psi_{0}\right)$. Due to our parametrization this means

$$
\psi_{l}(t+\delta t)-\psi_{l}(t)
$$

is large even for small $\delta t$.

Through (4) the result above, for each factor $j$, is injected into the time evolution of the fields. This leads to sensitive time dependence. ( See however the remarks in Sec.7.)

The overall phase of $g$ (for any $r$ ) does not contribute directly to the action density, expressible in terms of $g \bar{g}$ and its derivatives (Sec.2). But the amplitude at each order of iteration involves the phases of the lower ones (App). Moreover the phase is directly involved in the power series expansion in $r$.

The phase can be studied directly and analogously for any value of $r$ (inside the disc). The emphasis on the phase at $r=0$ is due to two reasons.

(a) The relative simplicity for $r=0$ permits a transparent derivation of the crucial properties.

(b) For spherical symmetry, in our ansatz, the time dependence is damped exponentially with increasing $r$ ( going toward the centre of the disc). Hence a small sphere around the origin in $R^{3}$ is the most suitable domain for studying the time evolution. The leading term of the action density for small $r$ is given explicitly (Sec.2) to display the role of the iterated phases.

(2). We do not ignore however other remarkable features associated with certain values of $r$ away from the spatial origine. ( The choice of the parameters $a$ and $k$ determines how far away or how close.) These are the domains on which the $Z^{(j)}$ 's can vanish and hence also $g$. They lead to discontinuous rotation numbers associated to the phases (App). So far as the zeros of $g$ can be located their cumulative effects lead to staircase like patterns. An example is given in Sec.2.

The rotation numbers are asymptotic quantities $(n \rightarrow \infty$ in (A58)). Hence discontinuities can arise even if we are dealing with well-behaved, integrable action densities. 
In [10] it is emphasized ( p.20), in the context of standard circle maps, that discontinuous rotation numbers can arise for smooth maps. Here they arise in the context of "annular" maps (App) when the annulus can become a disc. It is also closely related to the central property of our map that after each iteration "on the average" the phase turns twice as fast (App).

In Sec.2 the construction of the periodic fields is reformulated, with respect to our previous papers, in order to display prominently the role of the iterative map. The selfdual solutions are characterized by two topological integers. One is "instanton-like" $\left(P_{T}\right)$ and the other is "monopole-like" $(q)$. Their remarkable combined role in index theorems with $R^{3} \times S^{1}$ as the base-manifold for periodic fields $[2,4,6,7]$ are recapitulated in the context of explicit construction of spinor solutions (Sec.4).

Some special features of quasiperiodic fields are studied in Sec.3. For periodic backgrounds a finite number of normalizable, zero-mass spinor solutions are obtained by imposing on them (anti)periodic boundary conditions in $t$ (Sec.4). For quasiperiodic backgrounds the number cannot thus be limited, unless rational approximations of the component periods are considered. Nevertheless the spinor solutions are constructed in a way that works for both classes. The aim ( not realized here ) is to study the effect on the spinors of several mutually incommensurable periods in the background field.

For some particularly simple systems the possible effects of quasiperiodic kicks have been studied by several authors with different conclusions. ( See [11] and sources cited there.)

In a more general context ( quasiperiodic state with $k$ frequencies and a quasiperiodic attractor for the value $\mu=\mu_{0}$ of a continuous bifurcation parameter ) the situation has been summed up as follows ([12], p.631)

"For $k \geq 3$, strange attractors and positive characteristic exponent may be present for $\mu$ arbitrarily close to $\mu_{0}$."

We provide ( though only for zero mass and Euclidean signature ) exact, explicit solutions for spinors in four dimensions in a gauge field background that can bring into play an arbitrary number of mutually incommensurable periods. This can provide an interesting starting point for further investigations.

Our iterations can also be implemented in propagators (Sec.5). This can lead to a systematic semiclassical development for our classes of background.

Several directions are indicated (Sec.6) for possible generalizations of our study. The possibilities mentioned are - breaking spherical symmetry, mag- 
netic charge $q \geq 1$, gauge group $S U(N)$ with $N>2$ and the use of hyperbolic coordinates.

After presenting our formalism in full, certain general questions are taken up in the concluding remarks (Sec.7).

\section{A class of periodic selfdual gauge fields:}

The class we consider, to start with, has spherical symmetry in $R^{3}$ and periodicity in Euclidean time. The gauge group is $S U(2)$. Let $(t, r, \theta, \phi)$ be the standard time and radial coordinates with

$$
d s^{2}=d t^{2}+d r^{2}+r^{2}\left(d \theta^{2}+(\sin \theta)^{2} d \phi^{2}\right)
$$

Let $\left(\sigma_{r}, \sigma_{\theta}, \sigma_{\phi}\right)$ denote the projections of the Pauli matrices respectively on the directions indicated. The gauge potentials are given by

$$
\begin{gathered}
A_{r} \pm i A_{t}= \pm i\left(\partial_{r} \pm i \partial_{t}\right) \zeta \frac{\sigma_{r}}{2} \\
A_{\theta} \pm i(\sin \theta)^{-1} A_{\phi}= \pm i\left(e^{\zeta}-1\right)\left(\frac{\sigma_{\theta} \pm i \sigma_{\phi}}{2}\right)
\end{gathered}
$$

where

$$
e^{\zeta}=\frac{r}{(1-g \bar{g})}\left(\left(\partial_{r}^{2}+\partial_{t}^{2}\right)(g \bar{g})\right)^{\frac{1}{2}}
$$

Here $g$ is a holomorphic function $g(r+i t)$ in the $(r, t)$ half-plane $(r \geq 0)$, postulated to satify the following properties:

(1) $g \bar{g}=1+O(r)$ for $r \rightarrow 0$

(2) $g$ has no poles for $r \geq 0$

(3) $g$ falls exponentially as $r \rightarrow \infty$ (giving a constant logarithmic derivative)

(4) $g$ is periodic in $t$.

We start with strict periodicity. Quasiperiodicity will be defined and studied in Sec.3. Our previous studies of periodic instantons ([1]to [8]) will be reformulated below to display at each stage the role of the iterative map (App). 


\subsection{First iteration: (from monopoles to periodic in- stantons)}

The simplest choice satisfying all the constraints is evidently

$$
g=e^{-k(r+i t)} \quad(k>0)
$$

But now $g \bar{g}$ has no time dependence and from (7)

$$
e^{\zeta}=\frac{k r}{\sinh k r}
$$

One obtains the famous selfdual BPS monopole with the magnetic topological winding number

$$
q=1
$$

Here we have, of course, the Euclidean version, $A_{t}$ replacing the Higgs field.

In (8) $g$ is precisely $Z_{0}$ the initial point of the iterative map studied in App. Apply one iteration. Then

$$
g=\frac{a_{0}+e^{-k(r+i t)}}{\bar{a}_{0}+e^{k(r+i t)}}
$$

There is a spectacular change. One has now an authentic periodic solution characterized by two topological integers:

(1) The magnetic number remains unchanged since as $r \rightarrow \infty$ there is no essential change in the configuration. One has still the (monopole-like) number

$$
q=1
$$

(2) A second (instanton-like) topological integer $P_{T}$ is now given by the total action $S_{T}$ over one period $\left(T=2 \pi k^{-1}\right)$ divided by $8 \pi^{2}$. One defines

$$
8 \pi^{2} P_{T}=4 \pi \int_{0}^{T} d t \int_{0}^{\infty} d r\left(\partial_{r}{ }^{2}+\partial_{t}{ }^{2}\right) \omega
$$

where

$$
\begin{gathered}
\omega=\frac{1}{2} e^{2 \zeta}+\ln \left(\frac{1-g \bar{g}}{r}\right) \\
\left(\partial_{r}{ }^{2}+\partial_{t}{ }^{2}\right) \omega=\frac{1}{2}\left(\partial_{r}{ }^{2}+\partial_{t}{ }^{2}\right)\left(e^{2 \zeta}-2 \zeta\right)=\frac{1}{2}\left(\partial_{r}{ }^{2}+\partial_{t}{ }^{2}\right) e^{2 \zeta}+\frac{1}{r^{2}}\left(1-e^{2 \zeta}\right)
\end{gathered}
$$


For (9) one obtains

$$
P_{T}=2
$$

The computation of the action ill be given below in a form particularly suited to our purpose. But first let us introduce a more general form of $g$.

In (8) set, assuming $k$ to be sufficiently large,

$$
k=\sum_{j=1}^{n} k_{j} \quad\left(k_{j}>0\right)
$$

At this stage the $k_{j}$ 's are supposed to be mutually commensurate. Thus ,in evident notations,

$$
g=\prod_{j=1}^{n} g_{j}^{(0)}=\prod_{j=1}^{n} e^{-k_{j}(r+i t)}
$$

Now apply one iteration to each factor giving

$$
g=\prod_{j=1}^{n} g_{j}^{(1)}=\prod_{j=1}^{n}\left(\frac{a_{0}^{(j)}+e^{-k_{j}(r+i t)}}{\bar{a}_{0}^{(j)}+e^{k_{j}(r+i t)}}\right)
$$

In the notation of App

$$
g_{j}^{(1)}=Z_{1}^{(j)}
$$

Each factor now has its own period

$$
T_{j}=2 \pi k_{j}^{-1}
$$

Since the $k_{j}$ 's are mutually commensurate one can set

$$
k_{j}=\hat{k} \frac{P_{j}}{Q_{j}}
$$

where $P_{j}, Q_{j}$ are integers without common factor. Thus there is an overall period

$$
T=\frac{2 \pi}{\hat{k}}\left(\prod_{j} Q_{j}\right)
$$

We now compute the total action over T. Using (10) and Stoke's theorem with the boundary indicated by the limits one obtains

$$
S_{T}=4 \pi \int_{\delta}^{R} d r\left[\partial_{t} \omega\right]_{0}^{T}+4 \pi \int_{0}^{T} d t\left[\partial_{r} \omega\right]_{\delta}^{R} \quad(\delta \rightarrow 0, R \rightarrow \infty)
$$


The first integral vanishes due to periodicity. In the second one non-zero contributions come only from the limit $r \rightarrow 0$. These can be evaluated by using standard integrals $[3,4]$. But the very first step $(p=0)$ of our study (App) of the circle map and the small-r expansion furnishes the result directly.

From (12) and (A.38) as $r \rightarrow 0$,

$$
g \bar{g}=1+2\left(\sum_{j} \frac{d \psi_{1}^{(j)}}{d t}\right) r+2\left(\sum_{j} \frac{d \psi_{1}^{(j)}}{d t}\right)^{2} r^{2}+O\left(r^{3}\right)
$$

where

$$
e^{-i \psi_{1}^{(j)}}=\left(\frac{a_{0}^{(j)}+e^{-i k_{j} t}}{\bar{a}_{0}^{(j)}+e^{i k_{j} t}}\right)
$$

From $(A .19)$ as

$$
\begin{gathered}
k_{j} t=\psi_{0}^{(j)} \rightarrow \psi_{0}^{(j)}+2 \pi \\
\psi_{1}^{(j)} \rightarrow \psi_{1}^{(j)}+4 \pi
\end{gathered}
$$

Inserting $\omega$ of (10) in (16) and using (17), the only non-zero contributions are seen to come from

$$
\lim _{r \rightarrow 0}\left(\partial_{r} \ln \left(\frac{1-g \bar{g}}{r}\right)\right)=\sum_{j} \frac{d \psi_{1}^{(j)}}{d t}
$$

Hence, in terms of the previous definitions, one obtains quite simply

$$
\begin{aligned}
S_{T}= & 4 \pi\left(4 \pi \sum_{j=1}^{n} \frac{T}{T_{j}}\right)=8 \pi T\left(\sum_{j=1}^{n} k_{j}\right) \\
& =8 \pi^{2} 2\left(\prod_{j} Q_{j}\right)\left(\sum_{j=1}^{n} \frac{P_{j}}{Q_{j}}\right)
\end{aligned}
$$

Hence,

$$
P_{T}=2\left(\sum_{j=1}^{n} \frac{T}{T_{j}}\right)
$$

Since each $\left(\frac{T}{T_{j}}\right)$ is an integer $P_{T}$ is an even integer. Odd indices can be obtained through a simple modification described below. 


\subsection{Comments on the limiting values $a_{0}^{(j)}=0, \pm 1$ :}

In general the parameters $a$, will be assumed to satisfy $0<|a|<1$. The limits indicated are associated to monopoles and to standard aperiodic instantons. The following points are to be noted.

(1) For

$$
a_{0}^{(j)}=0, \psi_{1}^{(j)}=2 k_{j} t
$$

This limit, consistent with (19), can be taken smoothly. For all $a_{0}^{(j)}=0$ one has a static monopole. But having determined beforehand each $T_{j}$ and $T$, if this limit is taken a posteriori one obtains (formally) (22) in the simplest fashion.

(2) for

$$
a_{0}^{(j)}=1, \psi_{1}^{(j)}=k_{j} t
$$

As compared to (23) there is a rescaling by $\frac{1}{2}$. ( The choice -1 in (24) implies no essential change.) If say, only

$$
a_{0}^{(1)}=1
$$

one obtains, instead of (22),

$$
P_{T}=\frac{T}{T_{1}}+2\left(\sum_{j=2}^{n} \frac{T}{T_{j}}\right)
$$

Now $P_{T}$ can be odd. Consider, for example

$$
g=\left(\frac{\frac{1}{2}(1+\epsilon)+e^{-k(r+i t)}}{\frac{1}{2}(1+\epsilon)+e^{k(r+i t)}}\right) \prod_{j=1}^{n}\left(\frac{a_{j}+e^{-k(r+i t)}}{\bar{a}_{j}+e^{k(r+i t)}}\right)
$$

For

$$
\epsilon=-1, P_{T}=2 n+2 ; \quad \epsilon=1, P_{T}=2 n+1
$$

(3) For each $j$, taking the combined limit

$$
k_{j} \rightarrow 0, a_{0}^{(j)}=-1+k_{j} b_{j} \quad\left(b_{j}+\bar{b}_{j}>0\right)
$$

one obtains from (12)

$$
g=\prod_{j=1}^{n}\left(\frac{b_{j}-(r+i t)}{\bar{b}_{j}+(r+i t)}\right)
$$


This is Witten's multi-instanton solution [13] with centres on the time axis and total action on $R^{4}$

$$
S=8 \pi^{2}(n-1)
$$

The magnetic charge is lost in this limit.

To sum up, $g$ as given by (12), combines instanton-like and monopolelike aspects. It is a more general construction containing them as limits. As an expression of this double role such periodic selfdual configurations are characterized by the presence of two topological integers: one instanton-like $\left(P^{T}\right)$ and one monopole-like $(q)$. The combined role of these two in the index theorems will be discussed later (Sec.6). So far though $P_{T}$ can range through the entire spectrum of integers, $q$ is restricted to unity. This restriction can be removed (Sec.6).

Finally we just mention that concerning the rotation numbers defined in App, the result (A.59) implies for (12), which has $n$ factors, a staircase-like pattrn with $n$ steps. It was pointed in Sec.1 that jumps in the rotation numbers can arise though the action density remains smooth. We illustrate this explicitly for the simplest non-trivial case. Let

$$
g=\left(\frac{a+e^{-k(r+i t)}}{\bar{a}+e^{k(r+i t)}}\right)
$$

Here the crucial value of $r$ corresponding to (A.59) is

$$
e^{-k r}=a
$$

But now from (7),

$$
e^{2 \zeta}=(k r)^{2}\left((\sinh k r)^{-2}-\left(1-a^{2}\right)(\cosh k r+a \cos t)^{-2}\right)
$$

Inserting (33) in (10) one obtains a smooth action density.

The results (A.39) and (A.40) can be used to study the time dependence of the action density in a small sphere around $r=0$. We defer the discussion to be able to include higher iterations and quasiperiodicity.

\subsection{Higher iterations:}

For higher iterations one stays within the class of (quasi)periodic instantons with unit magnetic charge. But various interesting properties arise or are accentuated as the configuration becomes more complex. Some crucial features are presented below. 
A generalization of the ansatz (12) is automatically implemented. One more iteration on (9) gives (see (A.62))

$$
g=Z_{2}=Z_{0}^{-2}\left(\frac{a_{0}+Z_{0}}{\bar{a}_{0}+Z_{0}^{-1}}\right)\left(\frac{\mu_{+}+Z_{0}}{\bar{\mu}_{+}+Z_{0}^{-1}}\right)\left(\frac{\mu_{-}+Z_{0}}{\bar{\mu}_{-}+Z_{0}^{-1}}\right)
$$

where

$$
\mu_{ \pm}=\frac{1}{2}\left(\left(a_{0}+\overline{a_{0}} a_{1}\right) \pm\left(\left(a_{0}+\overline{a_{0}} a_{1}\right)^{2}-4 a_{1}\right)^{\frac{1}{2}}\right)
$$

The factor $Z_{0}^{-2}$ (i,e, $\left.e^{2 k(r+i t)}\right)$ is an increasing exponential in $r$, though $Z_{2}$ as a whole falls as $e^{-k r}$. This aspect is generalized by setting (compare (12))

$$
g=e^{l(r+i t)} \prod_{j=1}^{n}\left(\frac{a_{0}^{(j)}+e^{-k_{j}(r+i t)}}{\bar{a}_{0}^{(j)}+e^{k_{j}(r+i t)}}\right)
$$

with

$$
l<\left(\sum_{j=1}^{n} k_{j}\right)
$$

The total action, instead of (21), is now (assuming $l$ is so chosen that the overall period is still $T$ )

$$
S_{T}=4 \pi T\left(2 \sum_{j=1}^{n} k_{j}-l\right)
$$

Thus one has a lower action with the same number of parameters $a$. In (34) the factor $Z_{0}^{-2}$ lowers $S_{T}$ (and $P_{T}$ ) to give

$$
\left(8 \pi^{2}\right)^{-1} S_{T}=P_{T}=2(3-1)=4
$$

\subsection{Effect on rotation numbers:}

The rotation numbers are defined and discussed in App ((A.58) to (A.65)). In the course of time evolution of the gauge field generated by (34), $g$ can vanish for

$$
e^{-k r}=\left|a_{0}\right|,\left|\mu_{+}\right|,\left|\mu_{-}\right|
$$

though $\left|\mu_{+}\right|$and $\left|\mu_{-}\right|$may coincide to $\left|a_{1}\right|^{\frac{1}{2}}$. Thus though $S_{T}$ is doubled, the number of zeros are here (generically) tripled. 
Let $r_{1}<r_{2}<r_{3}$ be the three distinct roots of (38) in $r$ and let $\Omega_{2}$ denote the rotation number after the second iteration. Then (A.59) is generalized as follows.

$$
\frac{\Omega_{2}}{\Omega_{0}}=4, \frac{7}{2}, 3, \frac{5}{2}, 2, \frac{3}{2}, 1
$$

respectively for

$$
r<r_{1}, r=r_{1}, r_{1}<r<r_{2}, r=r_{2}, r_{2}<r<r_{3}, r=r_{3}, r>r_{3} .
$$

Cumulative effects of higher iterations will increase the number of steps. One can factorize $Z_{p+2}$ in terms of $Z_{p}$ analogously to (34). But rather than repeating such steps our aim is to show how the results of App can lead directly to remarkable properties. An example, in a different direction, follows.

\subsection{Action density near the spatial origin:}

Let us explore the role of the iterations in the action density in a small sphere about the origin where the intricate interplay of (quasi)periodic pulsations are least affected by exponential damping with increasing $r$. We generalize (12) by setting

$$
g=\prod_{j=1}^{n} Z_{p_{j}}^{(j)}
$$

where $Z_{p_{j}}^{(j)}$ is $e^{-k_{j}(r+i t)}$ iterated $p_{j}$ times. For each $p_{j}=1$ one obtains $(12)$. The doubling after each iteration of the contribution of each factor to the total action has to be taken into account. A simple particular case is given by (37). The general result is evident on noting how (A.19) generalizes (19) and hence the derivation of (21). For (40) one obtains, instead of (21),

$$
S_{T}=8 \pi T\left(\sum_{j=1}^{n} 2^{\left(p_{j}-1\right)} k_{j}\right)
$$

As the action increases, the "weight" of that particular field configuration in path integrals diminishes. Hence the interest of an extra factor, as in (35), bringing down the action as far as possible for a given period, as in (36). Having obtained the total action let us now take a close look at a small 
sphere around $r=0$. Using the results obtained in (A.38),(A.39) and (A.40) one obtains after simplifications for (40)

$$
e^{2 \zeta}=\frac{r^{2}}{(1-g \bar{g})^{2}}\left(\left(\partial_{r}{ }^{2}+\partial_{t}{ }^{2}\right)(g \bar{g})\right)=1-\frac{2}{3} \Gamma_{t} r^{2}+O\left(r^{3}\right)
$$

where

$$
\begin{array}{cc}
\Gamma_{t}= & \frac{1}{2} C^{2}+(\ddot{C} / C)-\frac{3}{2}(\dot{C} / C)^{2} \\
C= & \sum_{j=1}^{n} \dot{\psi}_{p_{j}}^{(j)} \equiv \dot{\Psi}
\end{array}
$$

Here the dots denote time derivatives and $\psi_{p_{j}}^{(j)}$ is the phase of the $\mathrm{j}$-th factor (for $r=0)$ in (40). This leads, for $\omega$ given by (10)

$$
\left(\partial_{r}{ }^{2}+\partial_{t}{ }^{2}\right) \omega=\frac{4}{3} \Gamma_{t} r^{2}+O\left(r^{3}\right)
$$

The total action over $R^{3}$ and a period $T$ is given by (41). Let $S(r)$ denote the action, at any instant $t$, integrated over a small sphere of radius $r$ about the origin. Then

$$
S(r)=4 \pi \int_{0}^{r} d r\left(\partial_{r}^{2}+\partial_{t}^{2}\right) \omega=\left(\frac{4}{3} \Gamma_{t}^{2}\right) V_{r}+\ldots
$$

where (the dots indicating higher powers of $r$ )

$$
V_{r}=\frac{4}{3} \pi r^{3}
$$

The leading term, logically, is proportional to the volume of the sphere.(This is rendered possible by the zero coefficient of the term linear in $r$ in (42).)

In (43) $\Gamma_{t}$ has an interesting structure. There is a "kinetic" term,

$$
\frac{1}{2} C^{2}=\frac{1}{2} \dot{\Psi}^{2}
$$

and the Schwarzian derivative of $\Psi$. For the simplest case (one factor with $p=1$ in (40)) using (A.17), with

$$
a=\left|a_{0}\right|, \chi=\psi_{0}-\alpha_{0}, \psi_{0}=k t
$$




$$
C=\dot{\Psi}=2 k \frac{1+a \cos \chi}{1+a^{2}+2 a \cos \chi} \equiv 2 k \frac{X}{Y}
$$

Hence

$$
\frac{\ddot{C}}{C}-\frac{3}{2}\left(\frac{\dot{C}}{C}\right)^{2}=k^{2} a\left(1-a^{2}\right)\left(\cos \chi+a(\sin \chi)^{2}\left(\frac{3}{2} X^{-1}+Y^{-1}\right)\right)(X Y)^{-1}
$$

This changes sign at points determined by the choice of $a_{0}$. But for all $a_{0}$, it is positive (negetive) for $\chi=0(\pi)$ respectively. For the general case (40) the very complex structure implied by (43) determines the time dependence.

\section{Quasiperiodicity:}

So far we have been considering strict periodicity, having just mentioned that the component periods $T_{j}$ need not necessarily be commensurate. We now take a closer look at this possibility.

As an example let $\left(k_{2}, k_{3}, \ldots, k_{n}\right)$ in $(12)$ be all mutually commensurate as in (14) but not with $k_{1}$. ( This relatively simple case will suffice to exhibit some remarkable features. It is possible to consider several incommensurate periods. One can also start with $Z_{0}$ given by (3) rather than by (2).)

Let $\hat{T}$ be the common overall period for $\left(T_{2}, T_{3}, \ldots, T_{n}\right)$, but not for $T_{1}$. One can take successive rational approximations of $\left(\frac{\hat{T}}{T_{1}}\right)$

$$
\left(\frac{\hat{T}}{T_{1}}\right)_{(\text {appr })}=\frac{N_{1}}{N_{2}}
$$

$N_{1}, N_{2}$ being integers without common factors. Then

$$
T=N_{2} \hat{T}
$$

is the overall period at that approximation. As the approximation is improved $N_{1}, N_{2}, T$ all increase without limit. ( Since there is no exact period, improving the approximation one tends to cover the entire time axis.) One can keep in mind the famous example of successive rational approximations of the "golden mean"

$$
G=\frac{1}{2}(\sqrt{5}-1)
$$


using the Fibonacci sequence. But our considerations will not be limited to any such particular case. For the $l$-th approximation, in evident notations, the action over the period $T^{(l)}$ is

$$
S_{l}=16 \pi^{2}\left(\frac{T^{(l)}}{T_{1}}+\sum_{j=2}^{n} \frac{T^{(l)}}{T_{j}}\right)
$$

As $l \rightarrow \infty$ so do $T^{(l)}$ and $S_{l}$. One can now consider the limiting form of the action per unit time or the "normalized" action

$$
S_{N}=\left(\frac{S_{l}}{T^{(l)}}\right)_{l \rightarrow \infty}
$$

This gives a continuous index, a positive real number, not an integer. The possible mathematical significance of $S_{N}$ has been discussed elsewhere [ 4,7]. The magnetic index $q$ does not vary in the successive stages described above. As one approaches the asymptotic $S_{2}$ in $R^{3}$ the exponential damping of time dependences give the same static configuration at each stage, namely that of the monopole. Hence our quasiperiodic instantons are characterized, not by two topological integers, but by one positive real number $\left(S_{N}\right)$ and one integer $(q)$. For the class of solutions under consideration $q=1$. More general possibilities are indicated in Sec.6.

So far we have been looking at quasiperiodic solutions from the point of view of successive approximations. Let us now look at some exact consequences of the postulated incommensurability.

In the case considered before, consider the Poincaré sections of the factor $g_{1}$ ( corresponding to the period $T_{1}$ ) for

$$
t=t_{0}+n T \quad(n=0,1,2, \ldots)
$$

One obtains

$$
g_{1}(n)=e^{-i 2 \alpha_{1}} \frac{\left|a_{1}\right|+e^{-k_{1}\left(r+i\left(t_{0}+n T\right)\right)+i \alpha_{1}}}{\left|a_{1}\right|+e^{k_{1}\left(r+i\left(t_{0}+n T\right)\right)-i \alpha_{1}}}
$$

We simplify notations by setting

$$
\begin{gathered}
a=\left|a_{1}\right|=a_{1} e^{i \alpha_{1}}, k_{1}=k, k \omega=k t_{0}-\alpha_{1} \\
X^{(n)}=e^{i 2 \alpha_{1}} g_{1}(n)=\frac{a+F_{n}}{a+F_{n}^{-1}}
\end{gathered}
$$


where

$$
F_{n}=e^{-k(r+i \omega+i n T)}=e^{-k(r+i \omega)-i 2 \pi n \delta}, \delta \neq \frac{N_{1}}{N_{2}} \quad\left(N_{1}, N_{2} \text { integers }\right)
$$

The irrationality of $\delta$ is the basic quasiperiodicity postulate. Now

$$
X^{(n+p)}=\frac{a+F_{n+p}}{a+F_{n+p}^{-1}}=\frac{a+F_{n} e^{-i 2 \pi p \delta}}{a+F_{n}^{-1} e^{i 2 \pi p \delta}}
$$

We show that if the parameters $\left(r, t_{0}, k, \alpha, n, p, \delta\right)$ are fine-tuned $X$ may come back just once to an initial value but no more (not even twice). From $(52)$

$$
F_{n}^{2}+e^{i 2 \pi p \delta} a\left(1-X^{(n+p)}\right) F_{n}-e^{i 4 \pi p \delta} X^{(n+p)}=0
$$

Eliminating $F_{n}$ from the equations for three distinct integer values of $p$ say,

$$
p=p_{1}, p_{2}, p_{3}
$$

one of which may be zero, one has as a necessary constraint the vanishing determinant

$$
\operatorname{det}\left|\begin{array}{ccc}
1 & a\left(1-X^{n+p_{1}}\right) e^{i 2 \pi p_{1} \delta} & -X^{n+p_{1}} e^{i 4 \pi p_{1} \delta} \\
1 & a\left(1-X^{n+p_{2}}\right) e^{i 2 \pi p_{2} \delta} & -X^{n+p_{2}} e^{i 4 \pi p_{2} \delta} \\
1 & a\left(1-X^{n+p_{3}}\right) e^{i 2 \pi p_{3} \delta} & -X^{n+p_{3}} e^{i 4 \pi p_{3} \delta}
\end{array}\right|=0
$$

For, say,

$$
X^{\left(n+p_{1}\right)}=X^{\left(n+p_{2}\right)}=X^{\left(n+p_{3}\right)}=X
$$

this reduces to

$X(1-X) e^{i\left(p_{1}+p_{2}+p_{3}\right) 2 \pi \delta}\left(\left(1-e^{i\left(p_{1}-p_{2}\right) 2 \pi \delta}\right)\left(1-e^{i\left(p_{2}-p_{3}\right) 2 \pi \delta}\right)\left(1-e^{i\left(p_{3}-p_{1}\right) 2 \pi \delta}\right)\right)=0$

The coefficient of $X(1-X)$ cannot vanish due to our quasiperiodicity postulate. Neither can $X^{(n+p)}$ be zero or unity for three distinct values of $p$. That would imply, for example for $X=1$,

$$
F_{n}^{2}=e^{i 4 \pi p_{j} \delta}
$$

for three different values of $p_{j}$ and so on. Hence the constraint (55) cannot be satisfied. So $X^{(n)}$ cannot come back twice exactly to a previous value. There is, however, no restriction concerning repeated very close approaches. 
Let us now look at the conditions necessary for a single return, namely

$$
X^{(n+p)}=X^{(n)}
$$

From (52) this is seen to imply

$$
\left(F_{n} e^{-i \pi p \delta}\right)^{2}+2 \lambda\left(F_{n} e^{-i \pi p \delta}\right)+1=0
$$

where

$$
\lambda=a^{-1} \cos \pi p \delta
$$

Hence

$$
\begin{gathered}
F_{n}=e^{i \pi p \delta}\left(-\lambda \pm \sqrt{\lambda^{2}-1}\right) \\
\text { Case(1) }:(|\lambda| \leq 1)
\end{gathered}
$$

Let $\lambda=\cos \eta \quad(\eta \mathrm{real})$. Then

$$
F_{n}=e^{-k(r+i \omega)-i 2 \pi n \delta}=-e^{i(\pi p \delta \pm \eta)}
$$

This implies $r=0$ and

$$
e^{-i\left(k t_{0}-\alpha_{1}+\pi(2 n+p) \delta \pm \eta\right)}=-1
$$

(The \pm sign implies one or the other. They do not hold simultaneously.)

$$
\text { Case }(2): \quad(|\lambda|>1)
$$

Let $\lambda=\operatorname{coth} \zeta$ ( $\zeta$ real). Then, retaining the solution leading to a real value of $r$,

$$
F_{n}=-(\tanh (\zeta / 2)) e^{i \pi p \delta}
$$

and

$$
e^{-k r}=|\tanh (\zeta / 2)|, \quad e^{-i\left(k t_{0}-\alpha_{1}+\pi(2 n+p) \delta\right)}= \pm 1
$$

It should be noted that $X^{(n+p)}=X^{(n)}$ does not imply a periodic situation. In the solutions above $n$ is not arbitrary for a given $p$. One can indeed show that given the foregoing situation

$$
X^{(n+2 p)} \neq X^{(n+p)}=X^{(n)}
$$


consistently with the impossibility of (55). In this context the ambiguity of sign of the inverse map (expressing $F_{n}$ in terms of $X_{n}$ ) plays an interesting role. But we will not present such details here.

Let us note one more point. The action density and the rotation numbers now involve mutually incommensurable numbers. It would be interesting to study in detail the time evolution of the coefficient (43)

$$
\begin{array}{cc}
\Gamma_{t}= & \frac{1}{2} C^{2}+(\ddot{C} / C)-\frac{3}{2}(\dot{C} / C)^{2} \\
C= & \sum_{j=1}^{n} \dot{\psi}_{p_{j}}^{(j)} \equiv \dot{\Psi}
\end{array}
$$

with three or more incommensurable periods (namely $k_{j}$ 's at the origin of the $\psi_{j}$ 's) varying the parameters $a$. Our purpose in leaving the period $T$ and $\Omega_{0}$ in (A.58) is now evident. In general one will have, to start with, incommensurable $\Omega_{0}^{(j)}$ 's corresponding to different factors of $g$. For the simple case considered above (only $k_{1}$ incommensurable with the rest and with $F_{n}$ corresponding to a section of $Z_{0}^{(1)}$ ) one has

$$
\Omega_{0}^{(1)}=2 \pi \delta
$$

$\delta$ being irrational. The cumulative effects of several irrational numbers have to be taken into account in a more general case.

The different roles of rational and irrational rotation numbers are wellknown $[9,10]$. Here the rotations of the phases are considered in the context of annular maps (App). The "average" doubling of the phase (sufficiently near the origin) for each iteration is also specific to our case. Though we have demonstrated the existence of striking discontinuities, the role of the rotation numbers remains to be explored.

\section{Index theorems and spinor solutions:}

Again we start with the periodic case, defering a discussion of quasiperiodicity. For periodic gauge fields the base manifold is $R^{3} \times S^{1}$ rather than $R^{4}$ or $S^{4}$. The index theorems have to take into account the boundary effects induced by $S^{1}$. This has been discussed elsewhere $[4,6,7]$. Here we just mention that the number of zero modes of spinors in periodic backgrounds characterized by two topological integers $\left(P_{T}, q\right)$

$$
=P_{T}-q \text { for periodic spinors of isospin } \frac{1}{2}(\text { see }[2,4])
$$


$=4 P_{T}-2 q$ for periodic spinors of isospin 1 (see [7])

$=P_{T}$ for antiperiodic spinors of isospin $\frac{1}{2}$ (see $\left.[6]\right)$

(The background is, of course, still periodic for antiperiodic spinors.) We construct below the spinor solutions in a more general and systematic fashion than in the previous papers. This is facilitated by introducing a gauge transformation leading from the Witten-type gauge introduced in (5) and (6) to a (quasi)periodic generalization of 't Hooft or Jackiw -Nohl -Rebbi (JNR) type solutions $[2,4,6,7]$. Two historical references for the standard (aperiodic) solutions are $[13,14]$.

\subsection{Gauge transformation :}

We start with $A_{\mu}$ given by (5) and (6) and gauge transform by

$$
G(r, t)=e^{-i d(r, t) \sigma_{r} / 2}
$$

where

$$
e^{i d}=\left(\frac{1-g}{1-\bar{g}}\right)\left(\frac{\left(\partial_{r}-i \partial_{t}\right) g}{\left(\partial_{r}+i \partial_{t}\right) \bar{g}}\right)^{\frac{1}{2}}
$$

One obtains

$$
A_{\mu}^{\prime}=G^{-1} A_{\mu} G-i G^{-1} \partial_{\mu} G=\sigma_{\mu \nu} \partial_{\nu} \ln \Sigma
$$

where $\sigma_{10}=\sigma_{23}=\sigma_{1}$ (cyclic), and

$$
\Sigma=\frac{1}{2 r}\left(\frac{1+g}{1-g}+\frac{1+\bar{g}}{1-\bar{g}}\right)=\frac{1}{r} \frac{(1-g \bar{g})}{(1-g)(1-\bar{g})}
$$

satisfying , since $\left(\partial_{r}+i \partial_{t}\right) g=0=\left(\partial_{r}-i \partial_{t}\right) \bar{g}$,

$$
\square \Sigma=\left(\partial_{t}^{2}+\partial_{r}^{2}+\frac{2}{r} \partial_{r}\right) \Sigma=0
$$

Thus we have obtained the famous ansatz leading to 't Hooft or JNR solutions. But here it is being implemented in the context of magnetically charged (quasi)periodic solutions rather than that of standard aperiodic instantons.

The poles of $\Sigma$ play an essential role in the construction of spinor solutions. The passage from $g$ to $\Sigma$ maintains contact with the iterative map implemented for $\mathrm{g}$. But this raises the problem of displaying in an additive 
form the poles of $\Sigma$. The roots of $g=1$ are not ,in general, explicitly available. Genrally there are $2 n$ roots for (12) , all for $r=0$. The particular case, with $(0<a<1)$ and

$$
g=\left(\frac{a+e^{-(r+i t)}}{a+e^{(r+i t)}}\right)^{n}
$$

is fully treated in [7]. We display some typical features of the passage from $g$ to $\Sigma$ through simple examples, motivating the generalization to follow.

$\underline{\text { (1) }}$ For $g=e^{-k(r i t)}$

$$
\begin{gathered}
\frac{1+g}{1-g}=\operatorname{coth} \frac{1}{2} k(r+i t) \\
\Sigma=\frac{1}{2 r}\left(\operatorname{coth} \frac{1}{2} k(r+i t)+\operatorname{coth} \frac{1}{2} k(r-i t)\right) \\
=\sum_{l=-\infty}^{\infty} \frac{1}{k^{2}} \frac{1}{r^{2}+\left(t-k^{-1} 2 \pi l\right)^{2}}
\end{gathered}
$$

This is the periodic form of the BPS monopole, gauge equivalent with the still better-known static form. The action over one period $(T=2 \pi / k)$ can be considered, quite formally, to be $8 \pi^{2}$ (or $P_{T}=1$ ). (See the comments following (8) and (23).)

$\underline{(2)}$ For, with $(0<a<1)$,

$$
\begin{gathered}
g=\left(\frac{a+e^{-k(r+i t)}}{a+e^{k(r+i t)}}\right) \\
P_{T}=2,
\end{gathered}
$$

and

$$
\frac{1+g}{1-g}=\frac{1+a}{2} \operatorname{coth} \frac{1}{2} k(r+i t)+\frac{1-a}{2} \operatorname{coth} \frac{1}{2} k\left(r+i t-i \pi k^{-1}\right)
$$

(3) For

$$
\begin{gathered}
g=e^{-k(r+i t)}\left(\frac{a+e^{-k(r+i t)}}{a+e^{k(r+i t)}}\right) \\
P_{T}=3 \quad \text { The simplest case of (26) and (27)) }
\end{gathered}
$$


and

$\frac{1+g}{1-g}=\lambda_{1}^{2} \operatorname{coth} \frac{1}{2} k(r+i t)+\lambda_{2}^{2} \operatorname{coth} \frac{1}{2} k\left(r+i t-i c k^{-1}\right)+\lambda_{3}^{2} \operatorname{coth} \frac{1}{2} k\left(r+i t+i c k^{-1}\right)$

where

$$
\operatorname{cosc}=-\frac{1+a}{2} ; \lambda_{1}^{2}=\frac{1+a}{3+a}, \lambda_{2}^{2}=\lambda_{3}^{2}=\frac{1}{3+a}
$$

For our normalization a general consequence is

$$
\sum_{i} \lambda_{i}^{2}=1
$$

The number of coth terms in $\frac{1+g}{1-g}$ gives $P_{T}$ if each one has the same period, say $T$ and distinct singularities. A term with period $(T / m)$ contributes $m$ units to $P_{T}$.

(4) Once the roots of $g=1$ are obtained the residues at the poles give the $\lambda$ 's. But this is not strictly necessary for constructing the spinor solutions.

Consider a case with two different periods $\left(2 \pi k^{-1}, \pi k^{-1}\right)$, namely, $(0<$ $a<1)$ and

$$
g=\left(\frac{a+e^{-k(r+i t)}}{a+e^{k(r+i t)}}\right)\left(\frac{a+e^{-2 k(r+i t)}}{a+e^{2 k(r+i t)}}\right)
$$

Now

$$
P_{T}=2+2.2=6, \quad q=1
$$

The zeros of $g$ (and hence the jumps in rotation numbers) correspond to

$$
e^{-k r}=a, \sqrt{a}
$$

It is sufficient to note that $g=1$ for

$$
r=0 ; \quad e^{i t}= \pm 1,\left(-\frac{1}{2} \pm i \frac{\sqrt{3}}{2}\right),\left(\frac{1-a}{2} \pm i \sqrt{1-\left(\frac{1-a}{2}\right)^{2}}\right)
$$

One can then construct $\left(P_{T}-q\right)=5$ periodic and $P_{T}=6$ antiperiodic spinor solutions. The explicit solutions will follow. 
More generally, using real parameters $a_{p}$ at each iteration one obtains some simplifications. Thus, for example, iterating $g$ as a whole (with $0<$ $\left.a_{p}<1\right)$

$$
g_{p+1}=\frac{a_{p}+g_{p}}{a_{p}+g_{p}^{-1}}
$$

and

$$
\frac{1+g_{p+1}}{1-g_{p+1}}=-\left(\frac{1+a_{p}}{2}\left(\frac{1+g_{p}}{1-g_{p}}\right)+\frac{1-a_{p}}{2}\left(\frac{1-g_{p}}{1+g_{p}}\right)\right)
$$

Hence $g_{p+1}=1$ for $g_{p}= \pm 1$, consistently with the doubling of the periodic action. For real $a_{0}, a_{1}$ and

$$
\begin{gathered}
g_{0}=e^{-k(r+i t)} \\
g_{2}=1 \quad \text { for } r=0 ; \quad \sin \frac{k t}{2}=0, \cos \frac{k t}{2}=0, \pm \sqrt{\frac{1-a_{0}}{2}}
\end{gathered}
$$

\subsection{Generalization of $Z_{0}$ and $g$ :}

Having noted the relation between the structures of $g$ and $\Sigma$ one can now invert the procedure. Start with

$$
\begin{aligned}
\Sigma & =\frac{1}{2 r} \sum_{l=1}^{n} \lambda_{l}^{2}\left(\operatorname{coth} \frac{1}{2} k_{l}\left(r+i t-i c_{l}\right)+\operatorname{coth} \frac{1}{2} k_{l}\left(r-i t+i c_{l}\right)\right) \\
& =\frac{1}{2 r}\left(\frac{1+g}{1-g}+\frac{1+\bar{g}}{1-\bar{g}}\right)
\end{aligned}
$$

where convenient conventions are

$$
\sum_{l=1}^{n} \lambda_{l}^{2}=1 ; \quad k_{n}>k_{n-1}>\ldots>k_{2}>k_{1}>0
$$

Now

$$
g=\frac{\sum_{l=1}^{n} \lambda_{l}^{2}\left(e^{k_{l}\left(r+i t-i c_{l}\right)}-1\right)^{-1}}{\sum_{l=1}^{n} \lambda_{l}^{2}\left(1-e^{-k_{l}\left(r+i t-i c_{l}\right)}\right)^{-1}}
$$

Thus we have motivated the parametrization (3) of $Z_{0}$ (here $g$ ). One can verify that (76) satisfies all the constraints listed below (7). In particular, $g$ is a phase for $r=0$ and drops as $e^{-k_{1} r}$ as $r$ becomes large. The major interest 
is that now, even in presence of an arbitrary number of different periods (different $k$ 's) $g$ is directly adapted to the construction of spinor solutions to follow. The $\Sigma$ of (74) can easily be generalized to break spherical symmetry. But we defer such considerations to Sec.6.

To give a better idea of the relation of (76) to the previous factorized form we cosider again some simple examples. Different subclasses of the previous form will be found already in the simplest examples of (76).

We set $n=2 ; \quad c_{1}=c_{2}=0$ and we use below often $\lambda_{1}^{2}+\lambda_{2}^{2}=1$.

$\underline{\text { (1) }}$ For $k_{1}=k_{2}=k$

$$
g=e^{-k(r+i t)}
$$

More generally, for all $k$ 's and all $c$ 's equal one has the monopole solution.

$\underline{(2)}$ For $k_{1}=k, k_{2}=2 k$

$$
g=\frac{\lambda_{1}^{2}+e^{-k(r+i t)}}{\lambda_{1}^{2}+e^{k(r+i t)}}
$$

(3) For $k_{1}=k, k_{2}=3 k$

$$
g=e^{k(r+i t)}\left(\frac{a+e^{-k(r+i t)}}{\bar{a}+e^{k(r+i t)}}\right)\left(\frac{\bar{a}+e^{-k(r+i t)}}{a+e^{k(r+i t)}}\right)
$$

where $a=\frac{1}{2}\left(\lambda_{1}^{2}+i \lambda_{1} \sqrt{4-\lambda_{1}^{2}}\right) \quad\left(a \bar{a}=\lambda_{1}^{2}<1\right)$

(4) For $k_{1}=2 k, k_{2}=3 k$

$$
g=\left(\frac{a_{1}+e^{-k(r+i t)}}{\bar{a}_{1}+e^{k(r+i t)}}\right)\left(\frac{a_{2}+e^{-k(r+i t)}}{\bar{a}_{2}+e^{k(r+i t)}}\right)
$$

where respectively for

$$
\begin{gathered}
1>\lambda_{1}^{2}>\frac{1}{4}, \quad a_{1}=\bar{a}_{2}=\frac{1}{2}\left(1+i \sqrt{4 \lambda_{1}^{2}-1}\right) ; \\
\frac{1}{4}>\lambda_{1}^{2}>0, \quad a_{1}=\bar{a}_{1}=\frac{1}{2}\left(1+\sqrt{1-4 \lambda_{1}^{2}}\right), \\
a_{2}=\bar{a}_{2}=\frac{1}{2}\left(1-\sqrt{1-4 \lambda_{1}^{2}}\right) ;
\end{gathered}
$$




$$
\lambda_{1}^{2}=\frac{1}{4}, \quad a_{1}=a_{2}=\frac{1}{2} .
$$

For special values of the $k$ 's and the c's in (76) some poles can coincide, diminishing $P_{T}$ accordingly. The preceding examples, apart from leading to simple factorized expressions for $g$ also illustrate this point. One degeneracy (common pole for $r=0$, sinkt $=0$ ) diminishes $P_{T}$ by 1 in each case. This is consistently incorporated in the factorized forms of $g$. Thus for $k_{1}=2 k, k_{2}=$ $3 k$ one obtains $P_{T}=4$ instead of 5 . We will assume, in general,that the choice of parameters in $\Sigma$ imply distinct poles.

Spinor solutions for zero mass were constructed [2] directly in Witten type gauge. But since the counting of the number of zero modes (and hence comparison with the index theorems) is more transparent in the 't Hooft or JNR type gauge, we start by generalizing (74) as follws. Let

$$
A_{\mu}=\sigma_{\mu \nu} \partial_{\nu} \ln \Sigma
$$

where, with $\epsilon= \pm 1$,

$$
\begin{aligned}
\Sigma=\frac{1}{2}(1+\epsilon)+ & \frac{1}{2 r} \sum_{j=1}^{n} \lambda_{j}^{2}\left(\operatorname{coth} \frac{1}{2} k_{j}\left(r+i t-i c_{j}\right)+\operatorname{coth} \frac{1}{2} k_{j}\left(r-i t+i c_{j}\right)\right) \\
& =\frac{1}{2}(1+\epsilon)+\sum_{j=1}^{n} \sum_{l=-\infty}^{\infty} \frac{\lambda_{j}^{2}}{k_{j}^{2}} \frac{1}{r^{2}+\left(t-k_{j}^{-1} 2 \pi l\right)^{2}}
\end{aligned}
$$

The role of $\epsilon$ is interesting concerning the index theorems. For $\epsilon=1$ one has a 't Hooft-like form. It can be shown that on the asymptotic sphere in $R^{3}$ one has now a dipole like configuration rather than a monopole.( See the discussion in [6] and the sources cited there.) Hence for

$$
\epsilon=1, \quad q=0
$$

while for

$$
\epsilon=-1, \quad q=1 \text {. }
$$

However, for both values $(\epsilon= \pm 1)$, the periodic action is the same. For an overall period $T$ and integers $n_{j}$ such that

$$
T_{j}=2 \pi k_{j}^{-1}, \quad T=2 \pi k^{-1}, \quad k_{j}=n_{j} k
$$




$$
S_{T}=8 \pi^{2} \sum_{j} n_{j}
$$

This can be seen as a limiting case of the familiar ('t Hooft and JNR) solutions as follows. Start by retaining in (77) the poles covering an interval NT. Define, in evident notations,

$$
S_{T}=\left(N^{-1} S_{N T}\right)_{N \rightarrow \infty}
$$

The value of $S_{N T}$ is given by the very well-known results for the 't Hooft and JNR solutions leading to

$$
\begin{gathered}
S_{T}=\frac{8 \pi^{2}}{N}\left(N \sum_{j} n_{j}-(1-\epsilon) / 2\right)_{N \rightarrow \infty} \\
=8 \pi^{2} \sum_{j=1}^{n} n_{j}
\end{gathered}
$$

Thus for the periodic case, in contrast with the aperiodic one, the change from $\epsilon=-1$ to $\epsilon=1$ does not increase $P_{T}$ by 1 but diminishes $q$ by 1 . For $n=1$ one has quite a special case.Then for $\epsilon=-1$ one has a static monopole gauge transformed to a periodic form, whereas for $\epsilon=1$ the periodicity is authentic but $q=0$.

\subsection{Spinor solutions:}

Having thoroughly prepared the ground, we at last introduce the spinors. Here we consider $[2,6]$ only isospin

$$
I=\frac{1}{2}
$$

( See [7] for $I=1$.) We consider only zero mass spinors.

For our conventions only upper (positive) helicity spinors have normalizable solution. Separating this helicity $\left(\Psi_{U}\right)$ the Dirac equation reduces to

$$
\bar{\alpha}_{\mu}\left(i \partial_{\mu}-A_{\mu}\right) \Psi_{U}=0
$$

where

$$
\bar{\alpha}_{\mu}=\left(\tau_{0}, i \vec{\tau}\right), \quad \alpha_{\mu}=\left(\tau_{0},-i \vec{\tau}\right)
$$


(We denote space-time Pauli matrices by $\tau_{\mu}$ and those in the isospace by $\sigma_{\mu}$.) The isospin components are separated as

$$
\Psi_{U}=\left|\begin{array}{c}
\Psi_{U}^{(+)} \\
\Psi_{U}^{(-)}
\end{array}\right|, \quad I_{3} \Psi_{U}^{( \pm)}= \pm \frac{1}{2} \Psi_{U}^{( \pm)}
$$

Set

$$
\Psi_{U}^{( \pm)}=\Sigma^{\frac{1}{2}}\left|\begin{array}{l}
a_{ \pm} \\
b_{ \pm}
\end{array}\right|
$$

and define

$$
\begin{aligned}
u & =\frac{1}{2}\left(x_{3}+i x_{0}\right), & \bar{u} & =\frac{1}{2}\left(x_{3}-i x_{0}\right) \\
v & =\frac{1}{2}\left(x_{1}+i x_{2}\right), & \bar{v} & =\frac{1}{2}\left(x_{1}-i x_{2}\right)
\end{aligned}
$$

Formally, the solutions are given (see [6] and the sources cited there) by

$$
\begin{gathered}
a_{+}=\partial_{v}\left(\Sigma^{-1} H\right), \quad b_{+}=-\partial_{u}\left(\Sigma^{-1} H\right) \\
a_{-}=-\partial_{\bar{u}}\left(\Sigma^{-1} H\right), \quad b_{-}=-\partial_{\bar{v}}\left(\Sigma^{-1} H\right)
\end{gathered}
$$

where

$$
\square H=\left(\partial_{u} \partial_{\bar{u}}+\partial_{v} \partial_{\bar{v}}\right) H=0
$$

Normalizable solutions are obtained by matching the poles of $H$ with the zeros of $\Sigma^{-1}$.From (77) it is evident that there are an infinite number of such possible choices of $H$. A finite number is obtained by imposing suitable boundary conditions relating $\Psi_{U}(t)$ and $\Psi_{U}(t+T), T=2 \pi K^{-1}$ being the period.

For periodic spinors satisfying

$$
\Psi_{U}(t)=\Psi_{U}(t+T)
$$

set

$H_{m_{j}}=\frac{1}{2 r}\left(\operatorname{coth} \frac{1}{2} K\left(r+i\left(t-c_{j}\right)-i 2 \pi k_{j}^{-1} m_{j}\right)+\operatorname{coth} \frac{1}{2} K\left(r-i\left(t-c_{j}\right)+i 2 \pi k_{j}^{-1} m_{j}\right)\right)$

with $T_{j}=2 \pi k_{j}^{-1}$ and

$$
m_{j}=1,2, \ldots, n_{j} ; \quad k_{j}=n_{j} K ; \quad(j=1,2, \ldots, n)
$$


These provide all the normalizable zero modes. The total number is

$$
\left(\sum_{j=1}^{n} n_{j}\right)
$$

But they are not all necessarily independent since

$$
\frac{1}{n_{j}} \sum_{m_{j}=1}^{n_{j}} \operatorname{coth} \frac{1}{2} K\left(r \pm i\left(t-c_{j}-2 \pi k_{j}^{-1} m_{j}\right)\right)=\operatorname{coth} \frac{1}{2} k_{j}\left(r \pm i\left(t-c_{j}\right)\right)
$$

(This is probably most easily derived via the logarithmic derivative of the well-known product formula for sinhnx.) Hence

$$
\sum_{j} n_{j}^{-1} \lambda_{j}^{2}\left(\sum_{m_{j}} H_{m_{j}}\right)=\Sigma-\frac{1}{2}(1+\epsilon)
$$

Thus for $\epsilon=-1$, in an evident notation,

$$
\sum_{j} n_{j}^{-1} \lambda_{j}^{2}\left(\sum_{m_{j}} \Psi_{U}^{\left(m_{j}\right)}\right)=0
$$

There is no such constraint for $\epsilon=1$. Moreover, for both cases $(\epsilon= \pm 1)$, as explained before

$$
P_{T}=\left(\sum_{j=1}^{n} n_{j}\right)
$$

Thus in both cases, consistently with the index theorems stated at the beginning of this section, the number of zero modes

$$
=P_{T}-q
$$

Here $q$ is limited to zero and one. But, on the other hand, we have done much more than counting the number of possible solutions. They have been obtained explicitly. One can now study, to take only one example, the time evolution of such spinor densities.

For antiperiodic spinors (in periodic backgrounds)

$$
\Psi_{U}(t)=-\Psi_{U}(t+T)
$$


and the correct choice [6] for $H$ turns out to be

$H_{m_{j}}=\frac{1}{2 r}\left(\operatorname{cosech} \frac{1}{2} K\left(r+i\left(t-c_{j}\right)-i 2 \pi k_{j}^{-1} m_{j}\right)+\operatorname{cosech} \frac{1}{2} K\left(r-i\left(t-c_{j}\right)+i 2 \pi k_{j}^{-1} m_{j}\right)\right)$

The linear constraint (83) is now absent even for $q=1(\epsilon=-1)$. There is no "magnetic defect",no subtraction of $q$, and one obtains(consistently with the result stated at the beginning of this section but now via explicit constructions)

number of zero modes $=P_{T}$.

Static configurations can be considered as a limiting case of periodic ones (infinite period) but not of antiperiodic ones. The Dirac modes in a monopole background that introduce the $q$-subtraction as a boundary effect are not relevant for antiperiodic spinors.

\subsection{Spinors in quasiperiodic backgrounds:}

Consider now the case where in (77) the $k$ 's are not all mutually commensurable. Generalizing the approximation (48) to several component irrational ratios one may construct (anti)periodic spinor solutions at each level of rational approximation. As this approximation is improved $P_{T}$ (approx) and hence the number of spinor modes will diverge. There being no exact period for the gauge field one cannot limit the number of spinor modes by imposing (anti)periodic boundary conditions as before. But our previous solutions provide a subset of "typical" ones which permit a comparative study of time evolution of spinors in periodic and quasiperiodic backgrounds respectively. Consider,for example, the subset ( periodic and antiperiodic respectively for a periodic background) corresponding to

$$
\begin{aligned}
H_{j}^{(+)} & =\frac{1}{2 r}\left(\operatorname{coth} \frac{1}{2} k_{j}\left(r+i t-i c_{j}\right)+\operatorname{coth} \frac{1}{2} k_{j}\left(r-i t+i c_{j}\right)\right) \\
H_{j}^{(-)} & =\frac{1}{2 r}\left(\operatorname{cosech} \frac{1}{2} k_{j}\left(r+i t-i c_{j}\right)+\operatorname{cosech} \frac{1}{2} k_{j}\left(r-i t+i c_{j}\right)\right)
\end{aligned}
$$

Suppose we start with mutually commnsurable $k$ 's and then vary some of them( infintesimaliy or more) to introduce incommensurability and quasiperiodicity. $H_{j}^{( \pm)}$will continue to give solutions, normalizable over any rationally approximated period or over unit time( analogously to (50)). One can express the spinor densities 


$$
\left(\Psi_{U}^{( \pm)}\right)^{\dagger} \Psi_{U}^{( \pm)}, \quad\left(\Psi_{U}^{( \pm)}\right)^{\dagger} \vec{\tau} \Psi_{U}^{( \pm)}
$$

in terms of $a_{ \pm}, b_{ \pm}$and $\Sigma$. One can implement the constraints due to the harmonic property of $\Sigma$ and the $H$ 's and (for our present case) those due to spherical symmetry. Then it might be rewarding to follow the time evolution of the densities for different values(small or some other crucial ones) of $r$, particularly when there are three or more incommensurable $k$ 's. Such a study is, however, entirely beyond the scope of this paper.

Let us finally note that starting with (76) as $g_{0}$ and iterating with real parameters as in (72), namely

$$
g_{p+1}=\frac{a_{p}+g_{p}}{a_{p}+g_{p}^{-1}}
$$

the roots of $g_{0}=1$ will form a subset of those of $g_{p}=1$. Hence defining

$$
\Sigma_{p}=\frac{1}{2 r}\left(\frac{1+g_{p}}{1-g_{p}}+\frac{1+\bar{g}_{p}}{1-\bar{g}_{p}}\right)
$$

and still retaining the explicit expressions $(85),(86)$ for $H_{j}^{( \pm)}$obtained for $\Sigma_{0}$, one obtains an interesting subset of spinor modes for the background corresponding to $\Sigma_{p}$, for both cases (periodic and quasiperiodic). At the level of $g_{0}$ the space-time dependence can already be quite complex. With iterations this will become much more so. But we will still have explicit solutions whose evolutions can be studied.

Note that our spinor solutions (except periodic ones for $q=0$ ) fall off exponentially for large $r$. For antiperiodic ones this is more evident. But though the leading term (for $q=1$ ) in $\left(\frac{H_{j}}{\Sigma}\right)$ is constant for periodic spinors, the presence of derivatives introduce again exponential damping. For $q=0$ periodic spinor densities fall off as $r^{-4}$. We have already studied the action density of the gauge fields near the origin(Sec.2). One can now study our "exponentially confined" fermion densities near the origin in such backgrounds.

\section{Propagators:}

For gauge field backgrounds considered in the preceding sections the propagators for massless, isospin $\frac{1}{2}$ fields were given in [3]. For periodic backgrounds the (anti)periodic propagators were presented in explicitly summed 
up, closed forms. Our iterative map can be implemented in them through the functions $g(\bar{g})$ corresponding to the points $x$ and $y$ of the propagator $\Delta(x, y)$. We recapitulate briefly the results of [3], where other sources are cited.

Define

$$
\begin{gathered}
t=x_{0}, t^{\prime}=y_{0}, \quad r=\sqrt{\vec{x}^{2}}, r^{\prime}=\sqrt{\vec{y}^{2}} \\
\sigma_{r}=\frac{\vec{\sigma} \cdot \vec{x}}{r}, \quad \sigma_{r^{\prime}}=\frac{\vec{\sigma} \cdot \vec{y}}{r^{\prime}} \\
G=\frac{1+g(r+i t)}{1-g(r+i t)}, \quad G^{\prime}=\frac{1+g\left(r^{\prime}+i t^{\prime}\right)}{1-g\left(r^{\prime}+i t^{\prime}\right)} \\
\Sigma=\frac{1}{2 r}(G+\bar{G}), \quad \Sigma^{\prime}=\frac{1}{2 r^{\prime}}\left(G^{\prime}+\bar{G}^{\prime}\right)
\end{gathered}
$$

and

$$
\begin{aligned}
& -i 2 F\left(r, t ; r^{\prime}, t^{\prime}\right)= \\
& \quad \frac{\left(1-\sigma_{r}\right)\left(1+\sigma_{r^{\prime}}\right)}{\left(t-t^{\prime}\right)+i\left(r-r^{\prime}\right)}\left(G-G^{\prime}\right)-\frac{\left(1+\sigma_{r}\right)\left(1-\sigma_{r^{\prime}}\right)}{\left(t-t^{\prime}\right)-i\left(r-r^{\prime}\right)}\left(\bar{G}-\bar{G}^{\prime}\right) \\
& +\frac{\left(1-\sigma_{r}\right)\left(1-\sigma_{r^{\prime}}\right)}{\left(t-t^{\prime}\right)+i\left(r+r^{\prime}\right)}\left(G+\bar{G}^{\prime}\right)-\frac{\left(1+\sigma_{r}\right)\left(1+\sigma_{r^{\prime}}\right)}{\left(t-t^{\prime}\right)-i\left(r+r^{\prime}\right)}\left(\bar{G}+G^{\prime}\right)
\end{aligned}
$$

Let unprimed fields $\left(A_{\mu}\right)$ correspond to $x$ and primed ones $\left(A_{\mu}^{\prime}\right)$ to $y$. Let

$$
\tilde{\Delta}(x, y)=\Sigma^{-\frac{1}{2}} \frac{F}{4 \pi^{2}(x-y)^{2}} \Sigma^{-\frac{1}{2}}
$$

and

$$
D_{\mu}=\left(\partial_{\mu}+i A_{\mu}\right)\left(\partial_{\mu}+i A_{\mu}\right)
$$

Then

$$
-D^{2} \tilde{\Delta}(x, y)=\delta^{4}(x-y)
$$

Thus $\tilde{\Delta}$ gives the aperiodic propagator for massless scalar fields. For $G\left(G^{\prime}\right)$ periodic in $t\left(t^{\prime}\right)$ with a period $T$, say, the periodic and the antiperiodic propagators are respectively defined to be,

$$
\Delta_{+}(x, y)=\sum_{n=-\infty}^{\infty} \tilde{\Delta}\left(x_{0}+n T, \vec{x} ; y_{0}, \vec{y}\right)
$$




$$
\Delta_{-}(x, y)=\sum_{n=-\infty}^{\infty}(-1)^{n} \tilde{\Delta}\left(x_{0}+n T, \vec{x} ; y_{0}, \vec{y}\right)
$$

Define

$$
\begin{gathered}
V_{1}=\left(t-t^{\prime}\right)+i|\vec{x}-\vec{y}|, V_{2}=\left(t-t^{\prime}\right)-i|\vec{x}-\vec{y}| \\
V_{3}\left(\epsilon, \epsilon^{\prime}\right)=\left(t-t^{\prime}\right)+i\left(\epsilon r+\epsilon^{\prime} r^{\prime}\right), \quad\left(\epsilon, \epsilon^{\prime}= \pm 1\right)
\end{gathered}
$$

and

$$
\begin{aligned}
S_{+}\left(\epsilon, \epsilon^{\prime}\right) & =\frac{\pi}{T} \frac{\left(\left(V_{2}-V_{3}\right) \cot \left(\frac{\pi}{T} V_{1}\right)+\text { cyclic }\right)}{\left(V_{1}-V_{2}\right)\left(V_{2}-V_{3}\right)\left(V_{3}-V_{1}\right)} \\
S_{-}\left(\epsilon, \epsilon^{\prime}\right) & =\frac{\pi}{T} \frac{\left(\left(V_{2}-V_{3}\right) \operatorname{cosec}\left(\frac{\pi}{T} V_{1}\right)+\text { cyclic }\right)}{\left(V_{1}-V_{2}\right)\left(V_{2}-V_{3}\right)\left(V_{3}-V_{1}\right)}
\end{aligned}
$$

(The indices $\left(\epsilon, \epsilon^{\prime}\right)$ are implicit in $V_{3}$ on the right hand sides.) Then one obtains [3],

$$
\Delta_{ \pm}(x, y)=\frac{i\left(\Sigma \Sigma^{\prime}\right)^{-\frac{1}{2}}}{8 \pi^{2}}\left(\begin{array}{c}
\left(G-G^{\prime}\right) S_{ \pm}(1,-1)\left(1-\sigma_{r}\right)\left(1+\sigma_{r^{\prime}}\right) \\
-\left(\bar{G}-\bar{G}^{\prime}\right) S_{ \pm}(-1,1)\left(1+\sigma_{r}\right)\left(1-\sigma_{r^{\prime}}\right) \\
+\left(G+\bar{G}^{\prime}\right) S_{ \pm}(1,1)\left(1-\sigma_{r}\right)\left(1-\sigma_{r^{\prime}}\right) \\
-\left(\bar{G}+G^{\prime}\right) S_{ \pm}(-1,-1)\left(1+\sigma_{r}\right)\left(1+\sigma_{r^{\prime}}\right)
\end{array}\right)
$$

The propagator for spinors is obtained now through a standard prescription [15] as

$$
\left(\gamma \cdot D(x) \Delta(x, y)\left(1+\gamma_{5}\right) / 2+\Delta(x, y) \gamma \cdot \overleftarrow{D}(y)\left(1-\gamma_{5}\right) / 2\right)
$$

where $\Delta$ can be $\tilde{\Delta}, \Delta_{+}$or $\Delta_{-}$.

All that has been assumed is that $g$ (at $x, y$ or at any other point) saitfies the properties listed under (7). Thus $g$ can involve an arbitrary number of iterations. When $x$ and $y$ are both close to the origine, $G$ and $G^{\prime}$ will exhibit strongly and simultaneously the consequences of the chaotic aspects studied in App. (See the remarks in Sec.7.) For a quasiperiodic background one can consider the aperiodic $\tilde{\Delta}$ or $\Delta_{ \pm}$for some adequate rational approximation.

\section{Generalizations:}

Only brief indications will be given below concerning some possible generalizations. 


\subsection{Beyond spherical symmetry for $q=0,1$ :}

One can generalize (66) and (77) as follows. Let

$$
A_{\mu}=\sigma_{\mu \nu} \partial_{\nu} \ln \Sigma
$$

where, with $\epsilon= \pm 1$,

$$
\Sigma=\frac{1}{2}(1+\epsilon)+\sum_{m=1}^{M} \frac{1}{2 r_{m}}\left(G_{m}+\bar{G}_{m}\right)
$$

and $G_{m}\left(r_{m}+i t\right)$ is a holomorphic function with

$$
r_{m}=\left|\vec{x}-\vec{x}_{m}\right|
$$

Choosing

$$
G_{m}=\frac{1+g_{m}}{1-g_{m}}
$$

where $g_{m}$ is now given by (40) with the origin translated to $\vec{x}_{m}$, iterations can be introduced independntly for each centre. One can have a "gas" (dilute or dense) of (quasi)periodic instantons. In [7] spinors were studied in such a background without iterations. One can also start by generalizing (76) including shifts of origin, starting for the m-th term with

$$
g_{m}^{(0)}=\frac{\sum_{l=1}^{n_{m}} \lambda_{l, m}^{2}\left(e^{k_{l, m}\left(r_{m}+i t-i c_{l, m}\right)}-1\right)^{-1}}{\sum_{l=1}^{n_{m}} \lambda_{l, m}^{2}\left(1-e^{-k_{l, m}\left(r_{m}+i t-i c_{l, m}\right)}\right)^{-1}}
$$

Then one may apply iterations independently for each $m$. As explained in Sec.4 a subset of spinor solutions can readily be obtained.

\section{2 $q>1$; spherical symmetry necessarily broken:}

The ansatz (77) (or (96)) is not suitable for generalizing beyond $q>1$. This is one of our reasons for starting with (7). The general formulation of linear pairs [16] can thus be applied specifically $[1,4]$ to (quasi)periodic fields for constructing higher $q$ solutions. Details can be found in those papers. Here we just indicate where iterations can be implemented. 
For $q=2$, one starts with two functions

$$
\begin{aligned}
& g_{1}=\prod_{j=1}^{n}\left(\frac{a_{j}+e^{-k_{j}(R+i t)}}{\bar{a}_{j}+e^{k_{j}(R+i t)}}\right) \\
& g_{2}=\prod_{j=1}^{n}\left(\frac{b_{j}+e^{-k_{j}(\bar{R}+i t)}}{\bar{b}_{j}+e^{k_{j}(R+i t)}}\right)
\end{aligned}
$$

where, with real $c$,

$$
\begin{aligned}
& R=\left(r^{2}-c^{2}-i 2 c r \cos \theta\right)^{\frac{1}{2}} \\
& \bar{R}=\left(r^{2}-c^{2}+i 2 c r \cos \theta\right)^{\frac{1}{2}}
\end{aligned}
$$

implying an imaginary translation $(i c)$ parallel to the $z$-axis. For the solutions to be regular the parameters $\left(c, a_{j}, b_{j}\right)$ have to satisfy constraints $[1,4]$. More geneally one starts with $q$ functions $g$ for charge $q$. Iterations can be implemented for these functions. But one must then verify carefully the regularity constraints afterwards. That, presumabbly, would be difficult.

\subsection{Gauge group SU(N):}

Selfdual, (quasi)periodic solutions for arbitrary $N$ were presented in [5]. Again we only indicate, in the simplest case for $N>2$, namely $S U(3)$, how the $g$ functions( which can be iterated) appear in the class of solutions

obained. The details of the generalized ansatz [5] will not be reproduced here.

For $S U(3)$ we just note that, instead of one funtion $e^{\zeta}$, as in (5),(6) and (7), one needs two

$$
\begin{aligned}
& e^{-b_{1}}=d_{1} P^{-1}\left(r^{2}\left(\partial_{r}^{2}+\partial_{t}^{2}\right)(g \bar{g})\right)^{-1}\left(\left(p_{3}-p_{2}\right)(g \bar{g})^{p_{1}}+\text { cyclic }\right) \\
& e^{-b_{2}}=d_{2} P^{-1}\left(r^{2}\left(\partial_{r}^{2}+\partial_{t}^{2}\right)(g \bar{g})\right)^{-1}\left(\left(p_{3}-p_{2}\right)(g \bar{g})^{2-p_{1}}+\text { cyclic }\right)
\end{aligned}
$$

where $d_{1}, d_{2}$ are constants( given in [5]),

$$
P=\left(p_{2}-p_{1}\right)\left(p_{3}-p_{1}\right)\left(p_{3}-p_{2}\right)
$$

and $\left(p_{1}, p_{2}, p_{3}\right)$ are rational numbers satisfying

$$
p_{1}<p_{2}<p_{3} ; \quad p_{1}+p_{2}+p_{3}=3
$$


To avoid certain problems concerning branch points(explained in [5]) we set

$$
g=\left(\prod_{j=1}^{n}\left(\frac{a_{j}+e^{-k_{j}(r+i t)}}{\bar{a}_{j}+e^{k_{j}(r+i t)}}\right)\right)^{Q}
$$

such that $Q p_{i},(i=1,2,3)$ are integers.

Iterations can be introduced separately for the factors of $g$ or for $g$ as a whole. One can also use $g$ of (76) as a starting point.

For higher values of $N$ a set of $N-1$ independent parameters $p$ enters into the solutions [5]. For $N>2$ a single magnetic winding number is no longer sufficient for characterizing the asymptotic configurations in $R^{3}$. This is one reason for an increasing number of parameters.

\subsection{Use of hyperbolic coordinates:}

The uses of the coordinate transformation

$$
(r+i t)=\tanh \frac{1}{2}(\rho+i \tau)
$$

in the construction of instantons or the so called hyperbolic monopoles were studied in a series of papers. (Apart from [17] they are all summerized in the review article [18].) The metric is

$$
\begin{aligned}
d s^{2} & =d t^{2}+d r^{2}+r^{2}\left(d \theta^{2}+(\sin \theta)^{2} d \phi^{2}\right) \\
& =(\cosh \rho+\cos \tau)^{-2}\left(d \tau^{2}+d \rho^{2}+(\sinh \rho)^{2}\left(d \theta^{2}+(\sin \theta)^{2} d \phi^{2}\right)\right)
\end{aligned}
$$

In $[17,18] \tau$-static solutions( depending on $(r, t)$ through $\rho$ ) were considered. In $[2,4]$ we indicated the passage from the $t$-periodic to the $\tau$-periodic solutions. One can replace in $(6)$ the subscripts $(r, t)$ by $(\rho, \tau)$ respectively and set

$$
e^{\zeta}=\frac{\sinh \rho}{(1-g \bar{g})}\left(\left(\partial_{\rho}^{2}+\partial_{\tau}^{2}\right)(g \bar{g})\right)^{\frac{1}{2}}
$$

and, for example,

$$
g=\prod_{j=1}^{n}\left(\frac{a_{j}+e^{-k_{j}(\rho+i \tau)}}{\bar{a}_{j}+e^{k_{j}(\rho+i \tau)}}\right)
$$


The action is evaluated in [2]. To avoid irregularities at

$$
\rho=0, \tau= \pm \pi
$$

the $k$ 's in (103) have to be integers. ( The role of the conformal factor in (101) is crucial concerning this point [18].) This is a restriction. But the formalism is more general in the follwing sense. A simple rescaling

$$
\rho=\lambda r^{\prime}, \tau=\lambda t^{\prime} ; \quad A_{\tau}=\lambda^{-1} A_{t^{\prime}}, A_{\rho}=\lambda^{-1} A_{r^{\prime}}
$$

gives back the previous formalism, in the limit $\lambda \rightarrow 0$, with

$$
d s^{\prime 2}=4 \lambda^{-2} d s^{2}=d t^{\prime 2}+d r^{\prime 2}+r^{\prime 2}\left(d \theta^{2}+(\sin \theta)^{2} d \phi^{2}\right)
$$

The restriction on the $k$ 's can be lifted after the scaling limit is taken.

But let us consider the situation without any such rescaling. As it stands, $g$ in (103) has already a quite special type of $(r, t)$ dependence via $(\rho, \tau)$. After several iterations one can have a very complex $(r, t)$ dependence, say for the action density. But the situation can still be studied relatively simply using $(\rho, \tau)$.

The coordinate transformation introduced maps the $(r, t)$ half-plane on the strip

$$
0 \leq \rho<\infty, \quad-\pi \leq \tau \leq \pi
$$

More generally one can consider our solutions in the context of three noncompact and one compactified dimension, all with the same signature. If $T$ is the period associated with the last one then the condition concerning integer values, say $n_{j}$ of $k_{j}$ is to be generalized to $k_{j}=\left(2 \pi n_{j}\right) / T$ assuring single valued solutions. One can also consider the possibility of embedding our solutions into such subspaces of higher dimensional spaces.

\section{Remarks:}

Chaos in gauge theories is a popular topic. Many authors have studied various aspects of this domain. A convenient reference is [19], a book devoted to this field with a long bibliography. Among more recent papers one may note [20] again citing many sources. Compared to most of the above-mentioned studies, ours is more modest in one respect but more ambitious in another. 
We have shown (App) that our iterative map, though simple, is a chaotic one. But it's implementation upto any given order does not automatically render our field configurations fully chaotic. The precise way in which the implemented iterations influence the time evolution of the gauge field has been pointed out in Sec.1. When the configuration is strictly periodic it comes back, by definition, to it's initial state after each period T. But the sensitive time dependence discussed in Sec.1 implies that any quantity (such as the action density at a given point or within a small volume) can fluctuate, within a single period, more and more with higher number of iterations and in a more complex fashion. It can wander far and waywardly before coming back. When the configuration is quasiperiodic it does not return repeatedly to an inital state (Sec.3) though arbitrarily close approaches are possible. How should one precisely characterize such fields with increasingly sensitive time dependence generated by iterations? We have not adequately explored the implications, the consequences of the two most striking features of our solutions, namely sensitive time dependence and jumps in rotation numbers. We have exhibited their existence. A more thorough exploration can probably indicate a satisfactory characterization. At this stage, after a "large" number of iterations, "at the edge of chaos" might be a convenient description (though precaution is necessary due to broad, fashionable uses of this terminology). Sufficiently accurate numerical studies can help in understanding. But that is beyond the competence of the present author.

Having noted the limitations, one may now note the positive qualities of our approach. In order to be able to apply techniques develped for one dimentional dynamical systems authors frequently consider gauge fields depending, effectively, on one coordinate only. Thus, for example, one studies time evolutions of fields homogeneous in space ([21],[20] and a number of papers cited in [19]). In [22] the static problem is reduced to the one dimensional Duffing equation and then time dependence is introduced as a perturbation. It is known that, considred in full generality, Yang - Mills fields are non-integrable ([19] and sources cited there). The fully chaotic aspects are then related to this non-integrability.

Our approach, one may say, is antithetic to the preceding one. The intriguing features we exhibit via the mapping arise in fully integrable (explicitly solved) selfdual configurations. We start with solutions, found in our previous papers, which have a whole range of remarkable properties, quite apart from those revealed in the present study. They combine topological 
aspects of standard instantons and monopoles and are charactrized by two topological numbers (both integers for periodic solutions).Instead of considering solitonic and chaotic aspects to be entirely incompatible (and leaving it at that) we have tried to explore, using our mapping, how close and with what possible limitations, they can be brought together. This has revealed probably hitherto unsuspected possibilities.

Quark confinement has been studied using approaches as different as that of a dual superconductor and that of random fields (see Ch.11 of [19]). Our spinors (except for one subclass) provide explicit solutions damped exponentially away from the origin. This is not confinement but worth noting. It should also be noted that this damping increases with the temperature (with some typical frequency $K$, inverse of the period), while confinement is usually supposed to break down at a sufficiently high temperature. Spinors in backgrounds with several incommensurable frequencies should be further explored for a better understanding. Rather than being fully, dully chaotic they might provide a terrain fevourable to genesis of rewardingly complex patterns and sructures.

Periodic instantons have been considered from the beginning [23,24,25] to be of particular interest in the study of gauge fields at finite temperatures. Our more general solutions (as noted in Sec.1) show very clearly that strict periodicity involves extremely fine tuning of a set of paramters (the $k$ 's). Irrationals being dense on the real line, infinitesimal shifts in one or more $k$ 's can make a periodic solution quasiperiodic and vice versa. Since such solutions exist it would be quite artificial to consider periodic solutions exclusively. Numerically, as is well-known, it is a delicate task to distinguish between commensurable and incommensurable cases. But since their mathematical properties are strikingly different, can one understand better the significance, the role of quasiperiodicity in the context of finite temperature ? In a realistic situation the temperature cannot be absolutely steady. A suitably chosen interval covering small fluctuations of a roughly steady temperature would cover a continuum of frequencies. Slowly varying temperatures would again need different considerations. Though such aspects might be worth considering, we have no simple adequate answer concerning the role of quasiperiodicity in the context of finite temperatures. We have studied quasiperiodicity for the light they shed on topological aspects and the possibilities formally engendered by the presence of several incommensurable periods in the background. 
We have obtained the propagators for (quasi)periodic backgrounds in particularly convenient forms, where one can implement an arbitrary number of our iterations. One can next try to compute the fluctuation determinants. Then one can start to carry over the consequences of the chaotic features of our mapping into quantum domains through semi-classical developments. It would be interesting to see in what fashion and to what extent such features can thus seep through.

Our spinor solutuions are limited to zero mass and to the gauge group $S U(2)$. But they, along with the propagator for spinor fields (Sec.5), can provide a starting point, exploiting the full range of our selfdual solutions, for the study of quarks in a finite temperature gluon background.

I thank Pierre Collet for discussions concerning iterative maps. 


\section{Appendix}

An iterative map of the unit disc is presented and some of it's properties are studied. In Sec.1 we indicate how this mapping is implemented in the construction of (quasi)periodic gauge fields and with what consequences. See also the remarks in Sec.7.

\section{A.1 The map:}

Let $Z_{p}$ be a point in the unit disc, centered at the origin, in the complex plane. Consider the map, with $0<\left|a_{p}\right|<1$,

$$
Z_{p+1}=\frac{a_{p}+Z_{p}}{\bar{a}_{p}+Z_{p}^{-1}}=Z_{p} \frac{a_{p}+Z_{p}}{1+\bar{a}_{p} Z_{p}}
$$

For $0 \leq\left|Z_{p}\right| \leq 1$ one obtains $0 \leq\left|Z_{p+1}\right| \leq 1$

The inverse power in the denominator $\left(Z_{p}^{-1}\right)$ leads to properties quit different from those of the standard Möbius type maps. The most evident difference is that here zero is a fixed point. But there are other profound differences. To take just one example, the Schwarzian derivative (identically

zero for Möbius maps) makes an interesting contribution in the action density near the spatial origin( Sec.2). One may set

$$
a_{p}=a_{p-1}=\cdots=a_{0}=a
$$

A more general possibility is

$$
a_{p}=f\left(a_{p-1}\right)
$$

with $f$ so chosen as to guarantee $0<\left|a_{p}\right|<1$ for $0<\left|a_{0}\right|<1$. An interesting example of $f$ will be given below. But the explicit form of $f$ will usually be unspecified, leaving room for eventual different convenient choices.

The crucial role of a suitable $(r, t)$ parametrization is emphasized in Sec.1. For the choice $(2), \psi_{0}$ being the phase of $Z_{0}$,

$$
Z_{0}=\left|Z_{0}\right| e^{-i \psi_{0}}=e^{-k(r+i t)} ; \quad(k>0, r \geq 0,-\infty<t<\infty)
$$

For the gauge fields we are finally interested in the space-time dependence. But in App $(r, t)$ should be considered as convenient notations defining

$$
\ln \left|Z_{0}\right|=-k r, \quad \psi_{0}=k t
$$


If (3) is chosen for $Z_{0}$, some different notation, say $(\widehat{k r}, \widehat{k t})$, would be more appropriate. We will however continue to use in App $(r, t)$ as in (A.4), hoping that no confusion can arise. Moreover some of our comments will refer specifically to (2) or (A.3).

Define

$$
Z_{p}=\left|Z_{p}\right| e^{-i \psi_{p}}, a_{p}=\left|a_{p}\right| e^{-i \alpha_{p}}, \chi_{p}=\psi_{p}-\alpha_{p}
$$

Then from (A.1),

$$
\left|Z_{p+1}\right| e^{-i\left(\psi_{p+1}-2 \alpha_{p}\right)}=\left|Z_{p}\right| e^{-i \chi_{p}} \frac{\left|a_{p}\right|+\left|Z_{p}\right| e^{-i \chi_{p}}}{1+\left|a_{p}\right|\left|Z_{p}\right| e^{-i \chi_{p}}}
$$

\section{A.2 Moduli:}

One has

$$
\begin{gathered}
\left|Z_{p+1}\right|^{2}=\left|Z_{p}\right|^{2} \frac{\left|a_{p}\right|^{2}+\left|Z_{p}\right|^{2}+2\left|a_{p}\right|\left|Z_{p}\right| \cos \chi_{p}}{1+\left|a_{p}\right|^{2}\left|Z_{p}\right|^{2}+2\left|a_{p}\right|\left|Z_{p}\right| \cos \chi_{p}} \\
\equiv\left|Z_{p}\right|^{2}\left|\tilde{Z}_{p}\right|^{2}
\end{gathered}
$$

where

$$
\left(1-\left|\tilde{Z}_{p}\right|^{2}\right)=\frac{\left(1-\left|a_{p}\right|^{2}\right)\left(1-\left|Z_{p}\right|^{2}\right)}{1+\left|a_{p}\right|^{2}\left|Z_{p}\right|^{2}+2\left|a_{p}\right|\left|Z_{p}\right| \cos \chi_{p}} \quad \geq 0
$$

Thus for

$$
\left|Z_{p}\right|=0,1,<1 ; \quad\left|Z_{p+1}\right|=0,1,<\left|Z_{p}\right|
$$

respectively.

Hence not only is zero a fixed point, but the circumference of the disc $\left(\left|Z_{0}\right|=1\right.$ or $\left.r=0\right)$ is stable as a whole leading to circle maps to be studied in detail soon. For the inverse map, both the roots of the quadratic

$$
Z_{P}^{2}+\left(a_{p}-\bar{a}_{p} Z_{p+1}\right) Z_{p}=Z_{p+1}
$$

must correspond to $0<\left|Z_{p}\right|<1$ for $0<\left|Z_{p+1}\right|<1$. ( For $\left|Z_{p}\right| \geq 1,\left|\tilde{Z}_{p}\right| \geq 1$ and hence $\left|Z_{p+1}\right| \geq 1$.) These roots coincide for

$$
Z_{p+1}=-\left(\frac{a_{p}}{\bar{a}_{p}}\right)\left(\frac{1-\sqrt{1-\left|a_{p}\right|^{2}}}{1+\sqrt{1-\left|a_{p}\right|^{2}}}\right)
$$


to

$$
Z_{p}=-\left(\frac{a_{p}}{1+\sqrt{1-\left|a_{p}\right|^{2}}}\right)
$$

Thus starting with $\left|Z_{0}\right|<1$ ( or $\left.r>0\right),|Z|$ moves away under iterations towards the attractive fixed point $(Z=0)$. Generically two values of $Z_{p}$ (which may coincide) are mapped on a $Z_{p+1}$ with a lesser modulus. But instead of a stepwise migration towards the fixed point zero, the latter might be reached abruptly due to the following feature.

\section{A.3 Zeros:}

One has $Z_{p+1}=0$ for

$$
Z_{p}=0
$$

and for

$$
Z_{p}=-a_{p}
$$

The second possibility is worth further study. As will be seen later, the more general condition $\left|Z_{p}\right|=\left|a_{p}\right|$ provides crucial domains of discontinuities of rotation numbers associated to the phases. Moreover

$$
Z_{p}=Z_{p-1} \frac{a_{p-1}+Z_{p-1}}{1+\bar{a}_{p-1} Z_{p-1}}=-a_{p}
$$

furnishes an interesting context for exploring the consequences of different choices for $f$ in $a_{p}=f\left(a_{p-1}\right)$. One has

$$
\left(\frac{Z_{p-1}}{\sqrt{a}_{p}}\right)^{2}+2 \lambda_{p}\left(\frac{Z_{p-1}}{\sqrt{a}_{p}}\right)+1=0
$$

where

$$
\lambda_{p}=\left(2 \sqrt{a}_{p}\right)^{-1}\left(a_{p-1}+a_{p} \bar{a}_{p-1}\right)
$$

Hence

$$
Z_{p-1}=\sqrt{a}_{p}\left(-\lambda_{p} \pm \sqrt{\lambda_{p}^{2}-1}\right)
$$

The two roots coincide to $Z_{p-1}=\mp \sqrt{a}_{p}$ for $\lambda_{p}= \pm 1$ or

$$
\sqrt{a}_{p}= \pm\left(\bar{a}_{p-1}\right)^{-1}\left(1-\sqrt{1-\left|a_{p-1}\right|^{2}}\right)
$$


Denoting, with real $\mu_{p}$,

$$
a_{p}=\left(\tanh \mu_{p}\right) e^{-i \alpha_{p}}
$$

(A.12) gives

$$
\tanh \mu_{p}=\left(\tanh \frac{1}{2} \mu_{p-1}\right)^{2}, \quad \alpha_{p}=2 \alpha_{p-1}
$$

This is an example of the choice of $f$ assuring special properties (here double zeros). For comparison note that choosing, for all $p$,

$$
\begin{gathered}
a_{p}=\bar{a}_{p}=a, \quad(0<a<1) \\
\lambda_{p}=\frac{1}{2} \sqrt{a}(1+a)=\cos \zeta
\end{gathered}
$$

say, $\zeta$ being real. Now (A.11) gives

$$
Z_{p-1}=-\sqrt{a} e^{\mp i \zeta}
$$

\section{A.4 Circle map on the circumference (chaotic aspects):}

For $\left|Z_{0}\right|=1$, (i.e. $\left.r=0\right)$, for all $p$,

$$
\left|Z_{p}\right|=1 .
$$

For the phases $\psi_{p}$, using the notations of (A.5), one has the circle map

$$
e^{-i \psi_{p+1}}=\frac{a_{p}+e^{-i \psi_{p}}}{\bar{a}_{p}+e^{i \psi_{p}}}=e^{-i 2 \alpha_{p}} \frac{\left|a_{p}\right|+e^{-i \chi_{p}}}{\left|a_{p}\right|+e^{i \chi_{p}}}
$$

Hence

$$
\begin{aligned}
& \frac{d \psi_{p+1}}{d \psi_{p}}=2\left(\frac{1+\left|a_{p}\right| \cos \chi_{p}}{1+\left|a_{p}\right|^{2}+2\left|a_{p}\right| \cos \chi_{p}}\right) \\
& =1+\frac{\left(1-\left|a_{p}\right|^{2}\right)}{\left(1-\left|a_{p}\right|\right)^{2}+4\left|a_{p}\right|\left(\cos \left(\chi_{p} / 2\right)\right)^{2}} \quad>1
\end{aligned}
$$

Thus $\psi_{p+1}$ is monotonic (increasing) in $\psi_{p}$ and

$$
\begin{aligned}
e^{-i\left(\psi_{p+1}-2 \alpha_{p}\right)} & =1 \quad \text { for } \quad \chi_{p}=0, \pi, 2 \pi \\
& =-1 \quad \text { for } \quad \cos \chi_{p}=-\left|a_{p}\right|, \quad \sin \chi_{p}= \pm \sqrt{1-\left|a_{p}\right|^{2}}
\end{aligned}
$$


Hence as

$$
\begin{gathered}
\psi_{p} \rightarrow \psi_{p}+2 \pi \\
\psi_{p+1} \rightarrow \psi_{p+1}+4 \pi
\end{gathered}
$$

(This result also follows from an approach analogous to the one leading to (A.52) for $\left|Z_{0}\right|>\left|a_{0}\right|$, since here $\left|Z_{p}\right|=1>\left|a_{p}\right|$. But the foregoing instructive one follows the rotations in more detail.) The result (A.19) is fudumental in computing the actions of the gauge fields after iterations (Sec.2). But here we concentrate on another aspect.

It is well-known ([9], p.50 and also p.18) that the apparently very simple circle map

$$
\theta_{p+1}=2 \theta_{p}, \quad \frac{d \theta_{p+1}}{d \theta_{p}}=2
$$

is chaotic. It satisfies all the requisite conditions, the most important one being a srtong sensitiveness to initial conditions (here in the form of expansiveness with index $\ln 2$ ). Our example (A.16) will be seen to satisfy the same conditions, but in a subtler fashion. In fact, our case contains (A.20) as a particularly simple limit $\left(a_{p}=0\right)$. "On the average" $\psi_{p+1}$ turns twice as fast as $\psi_{p}$. But the rate is less than twice in one domain and just sufficiently more than twice in the complementary one to compensate.

For

$$
\begin{aligned}
\cos \chi_{p} & = \pm 1 \\
v_{p} \equiv \frac{d \psi_{p+1}}{d \psi_{p}} & =\frac{2}{1 \pm\left|a_{p}\right|}
\end{aligned}
$$

and for

$$
\cos \chi_{p}=-\left|a_{p}\right|, \quad v_{p}=2
$$

The complementary domains are

$$
\begin{gathered}
1 \geq \cos _{p}>-\left|a_{p}\right| \quad\left(v_{p}<2\right) \\
-1 \leq \cos \chi_{p}<-\left|a_{p}\right| \quad\left(v_{p}>2\right)
\end{gathered}
$$

As $\left|a_{p}\right| \rightarrow 1$ the first domain increases, but so does $v_{p}$ in the other to compensate (becoming very high near $\cos \chi_{p}=-1$ ). 
After $p$ iterations (with $\psi_{0}=k t$ )

$$
\begin{aligned}
\frac{d \psi_{p}}{d \psi_{0}} & =\frac{1}{k} \frac{d \psi_{p}}{d t}=\prod_{j=0}^{p-1} v_{j} \\
& =\prod_{j=0}^{p-1}\left(\frac{2\left(1+\left|a_{j}\right| \cos \chi_{j}\right)}{1+\left|a_{j}\right|^{2}+2\left|a_{j}\right| \cos \chi_{j}}\right)
\end{aligned}
$$

and

$$
\frac{d^{2} \psi_{p}}{d \psi_{0}^{2}}=\frac{1}{k^{2}} \frac{d^{2} \psi_{p}}{d t^{2}}=\frac{d \psi_{p}}{d \psi_{0}}\left(\sum_{j=0}^{p-1} V_{j}\left(\frac{d \psi_{j}}{d \psi_{0}}\right)\right)
$$

where

$$
V_{j}=\frac{\left(1-\left|a_{j}\right|^{2}\right)\left|a_{j}\right| \sin \chi_{j}}{\left(1+\left|a_{j}\right| \cos \chi_{j}\right)\left(1+\left|a_{j}\right|^{2}+2\left|a_{j}\right| \cos \chi_{j}\right)}
$$

In (A.22) each factor $v_{j}>1$. But due to the factor $\sin \chi_{j}$ in $V_{j}$ the terms in (A.23) can change sign.

The sensitive dependence on initial data should be evident from the preceding analysis. But let us formulate it more precisely in terms of a characteristic index. For (A.20) the index is evidently $\ln 2$ [9]. This is recovered in our case in the limit of each $a_{j}=0$. For (A.16) it depends on the sequence $\left(a_{0}, a_{1}, \ldots\right)$. But it has a positive lower bound. In (A.22) replacing each $v_{j}$ by it's upper and lower bound respectively

$$
\prod_{j=0}^{N-1}\left(\frac{2}{1-\left|a_{j}\right|}\right) \geq \frac{d \psi_{N}}{d \psi_{0}} \geq \prod_{j=0}^{N-1}\left(\frac{2}{1+\left|a_{j}\right|}\right)>1
$$

Setting for simplicity all $\left|a_{j}\right|=|a|$, the characteristic index $\lambda$ satisfies

$$
\ln \left(\frac{2}{1-|a|}\right) \geq \lambda \geq \ln \left(\frac{2}{1+|a|}\right)>0
$$

More generally $\left(\frac{2}{1 \pm|a|}\right)$ should be considered as the geometric means of the corresponding products in (A.24).

For a map to be chaotic it must have a dense set of periodic points [9]. For (A.20) the periodic points are given by [9]

$$
\theta_{n}=2^{n} \theta=\theta+2 k \pi \quad \text { or } \quad \theta=\frac{2 k \pi}{2^{n}-1}
$$


where $k$ is an integer and

$$
0 \leq k \leq 2^{n}-1
$$

This is the situation for all $a$ 's zero in (A.16). But for (A.16) one cannot obtain as simply a general formula. One can however proceed stepwise to show how the periodic points remain dense but are shifted as the parameters $a$ incrase from zero. For simplicity consider all $a_{p}$ 's equal and real. Then

$$
e^{-i \psi_{p+1}}=\frac{a+e^{-i \psi_{p}}}{a+e^{i \psi_{p}}}
$$

gives

$$
\psi_{p+1}=2 \psi_{p}-2\left(\operatorname{asin} \psi_{p}-\frac{1}{2} a^{2} \sin 2 \psi_{p}+\ldots\right)
$$

Thus

$$
\psi_{n}=2^{n} \psi_{0}-a\left(\sum_{l=1}^{n} 2^{l} \sin 2^{n-l} \psi_{0}\right)+\mathrm{O}\left(a^{2}\right)
$$

Hence upto $\mathrm{O}(a)$ the periodic points are given by

$$
\psi=\frac{2 k \pi}{2^{n}-1}+a S_{1}
$$

where

$$
\begin{gathered}
S_{1}=\left(\frac{2^{n}}{2^{n}-1}\right)\left(\sum_{l=1}^{n} 2^{-n+l} \sin 2^{n-l}\left(\frac{2 k \pi}{2^{n}-1}\right)\right) \\
<\left(\frac{2^{n}}{2^{n}-1}\right)\left(\sum_{l=1}^{n} 2^{-n+l}\right)=2
\end{gathered}
$$

Thus the dense set (A.26) is shifted as shown above. One may now iterate to higher powers of $a$ and find an analogous situation. We cannot produce a general solution for (A.16) in a closed form, but the smooth continuity with (A.26) is clear enough.

A third criterion for chaoticity [9] is topological transitivity. This is satisfied by (A.16) as obviously as by (A.20). Our preceding analysis of rotations makes it evident that their effects can not remain confined in one particular segment of a circle.

Thus all the three criteria for being chaotic are satisfied by our map. 


\section{A.5 Series expansion near the circumference (small $r$ ):}

For the gauge field configurations principally considered in this paper the time dependence is exponentially damped as $r$ increases. So the (quasi)periodic time evolution is best studied for small $r$. For our mapping this corresponds to a domain near the cicumference at a distance $\left(1-e^{-k r}\right)$.

Let $\psi_{p}$ continue to denote the value of the phase for $r=0$ and let

$$
Z_{p}=e^{-i \psi_{p}}\left(1+C_{p}^{(1)} r+C_{p}^{(2)} r^{2}+\ldots\right)
$$

where the $C$ 's can be complex since the $r$-dependence of the total phase is included in them. The expansion (A.30) is general, but particularly useful for small $r$ due to evident reasons. ( Exceptionally, in this subsection only, $\psi_{p}$ denotes not the total phase but a part. The notation $\psi_{p}^{(0)}$ would have been more consistent. But this simplification, leaving room for other indices to come, should not cause confusion.)

Suppresing the index $p$ temporarily and using the holomorphy condition

$$
\left(\partial_{r}+i \partial_{t}\right) Z=0
$$

one obtains

$C^{(1)}+2 C^{(2)} r+3 C^{(3)} r^{2}+\ldots=-\frac{d \psi}{d t}\left(1+C^{(1)} r+C^{(2)} r^{2}+\ldots\right)-i\left(\frac{d C^{(1)}}{d t} r+\frac{d C^{(2)}}{d t} r^{2}+\ldots\right)$

Thus

$$
C^{(1)}=-\frac{d \psi}{d t}
$$

and, for $l>1$,

$$
l C^{(l)}=C^{(1)} C^{(l-1)}-i \frac{d C^{(l-1)}}{d t}
$$

The general solution is

$$
C^{(l)}=\frac{e^{i \psi}}{l !}\left(-i \frac{d}{d t}\right)^{l}\left(e^{-i \psi}\right)
$$

This expansion displays precisely how the chaotic properties of the circle map for the phase are carried over through $\psi$ and its derivatives in the coefficients. 
The result (A.34) is compact and elegant. But separate explicit expressions for the total phase and the amplitude of $Z$ are useful for the gauge fields. We give the first few tems of the $r$-expansion for both. The coefficients $\left(\psi^{(l)}, B_{l}\right)$ will now all be real. One obtains, keeping terms upto $\mathrm{O}\left(r^{4}\right)$,

$$
\begin{aligned}
Z & =e^{-i \psi}\left(1+C_{1} r+C_{2} r^{2}+C_{3} r^{3}+C_{4} r^{4}\right) \\
& =e^{-i\left(\psi^{(0)}+\psi^{(2)} r^{2}+\psi^{(4)} r^{4}\right)}\left(1+B_{1} r+B_{2} r^{2}+B_{3} r^{3}+B_{4} r^{4}\right)
\end{aligned}
$$

The coefficients $\psi^{(1)}$ and $\psi^{(3)}$ turn out to be zero and one obtains, in terms of the $C$ 's given before,

$$
\begin{aligned}
& \psi^{(2)}=\frac{i}{2}\left(C^{(2)}-\bar{C}^{(2)}\right) \\
& \psi^{(4)}=\frac{i}{2}\left(\left(C^{(4)}-\bar{C}^{(4)}\right)-\frac{1}{2}\left(\left(C^{(2)}\right)^{2}-\left(\bar{C}^{(2)}\right)^{2}\right)\right)
\end{aligned}
$$

and

$$
\begin{aligned}
B_{1} & =C^{(1)} \\
B_{2} & =\frac{1}{2}\left(C^{(2)}+\bar{C}^{(2)}\right) \\
B_{3} & =\frac{1}{2}\left(C^{(3)}+\bar{C}^{(3)}\right) \\
B_{4} & =\frac{1}{2}\left(C^{(4)}+\bar{C}^{(4)}\right)-\frac{1}{8}\left(\left(C^{(2)}\right)^{2}+\left(\bar{C}^{(2)}\right)^{2}\right)
\end{aligned}
$$

One may also note that

$$
Z \bar{Z}=1+D_{1} r+D_{2} r^{2}+D_{3} r^{3}+D_{4} r^{4}+\mathrm{O}\left(r^{5}\right)
$$

where

$$
\begin{aligned}
& D_{1}=2 C^{(1)} \\
& D_{2}=2\left(C^{(1)}\right)^{2} \\
& D_{3}=\frac{4}{3}\left(C^{(1)}\right)^{3}-\frac{1}{3} \frac{d^{2}}{d t^{2}} C^{(1)} \\
& D_{4}=\frac{2}{3}\left(\left(C^{(1)}\right)^{4}-C^{(1)} \frac{d^{2}}{d t^{2}} C^{(1)}\right)
\end{aligned}
$$


Higher order terms can be evaluated stepwise. These results hold, of course, for any $p$. If the product of several factors $\left(Z_{p} Z_{p^{\prime}}^{\prime} \ldots\right)$ is considered, one has the same expansion with

$$
C^{(1)}=-\frac{d}{d t}\left(\psi_{p}+\psi_{p^{\prime}}^{\prime}+\ldots\right)
$$

Consistency with this constraint is a useful check on the numerical coefficients obtained above. The notation indicates that for each factor the sequence of the parameters $a$, the periods involved and also the order of iterations can be different. The results (A.39) yield the leading term in the action density near the spatial origin (Sec.2).

\section{A.6 Annular maps and rotation numbers:}

We have studied some interesting proprties of our map on the circumference of the unit disc and nearby $(r=0, r \ll 1)$. One can continue an analogous study away from the edge. But we now concentrate on a different class of remarkable features associated to specific values of $r$ as it increases.

For $|Z|=1$ the iterations affect only the phase giving a circle map. For $|Z|<1$ the amplitude also changes. It diminishes and becomes a function of the phases of the previous steps. The domain of variation of $Z$ (as a function of these phases) becomes an annulus, which can, crucially, become a disc. To emphasize this aspect we use the term "annular map". The rotation numbers to be defined will be associated to the phases. We are fundumentally interested in variations of functions of the phase of $Z_{0}$ as the latter moves on a circle of radius $e^{-k r}$. this provides, most directly through (2), the link with the time evolution of gauge fields.

From (A.5) and (A.6), with the notations defined there $\left(\chi_{p}=\psi_{p}-\alpha_{p}, \ldots\right)$

$$
\left|Z_{p+1}\right|^{2}=\left|Z_{p}\right|^{2} \frac{\left|a_{p}\right|^{2}+\left|Z_{p}\right|^{2}+2\left|a_{p}\right|\left|Z_{p}\right| \cos \chi_{p}}{1+\left|a_{p}\right|^{2}\left|Z_{p}\right|^{2}+2\left|a_{p}\right|\left|Z_{p}\right| \cos \chi_{p}}
$$

and

$$
\psi_{p+1}=\psi_{p}+\frac{i}{2}\left(\ln f_{1}+\ln f_{2}\right)+2 \alpha_{p}
$$

where

$$
f_{1}=\left(\frac{\left|a_{p}\right|+\left|Z_{p}\right| e^{-i \chi_{p}}}{\left|a_{p}\right|+\left|Z_{p}\right| e^{i \chi_{p}}}\right), \quad f_{2}=\left(\frac{1+\left|a_{p}\right|\left|Z_{p}\right| e^{i \chi_{p}}}{1+\left|a_{p}\right|\left|Z_{p}\right| e^{-i \chi_{p}}}\right)
$$


In particular, for $p=0$ with $Z_{0}=e^{-k(r+i t)}$,

$$
\left|Z_{1}\right|^{2}=e^{-2 k r} \frac{\left|a_{0}\right|^{2}+e^{-2 k r}+2\left|a_{0}\right| e^{-k r} \cos \chi_{0}}{1+\left|a_{0}\right|^{2} e^{-2 k r}+2\left|a_{0}\right| e^{-k r} \cos \chi_{0}}
$$

For fixed $r$, this can be shown to be a monotonic increasing function of $\cos \chi_{0}$. One obtains

$$
\begin{aligned}
& \left|Z_{1}\right|_{\text {max }}=\left|Z_{0}\right|\left(\frac{\left|a_{0}\right|+\left|Z_{0}\right|}{1+\left|a_{0}\right|\left|Z_{0}\right|}\right) \\
& \left|Z_{1}\right|_{\text {min }}=\left|Z_{0}\right|\left(\frac{\left|a_{0}\right|-\left|Z_{0}\right|}{1-\left|a_{0}\right|\left|Z_{0}\right|}\right)
\end{aligned}
$$

The width of the annulus is

$$
W_{1}=\left|Z_{1}\right|_{\text {max }}-\left|Z_{1}\right|_{\min }
$$

For $\left|Z_{0}\right|>\left|a_{0}\right|$

$$
W_{1}=2\left|a_{0}\right|\left|Z_{0}\right|\left(\frac{1-\left|Z_{0}\right|^{2}}{1-\left(\left|a_{0}\right|\left|Z_{0}\right|\right)^{2}}\right)
$$

For $\left|Z_{0}\right|<\left|a_{0}\right|$

$$
W_{1}=2\left|Z_{0}\right|^{2}\left(\frac{1-\left|a_{0}\right|^{2}}{1-\left(\left|a_{0}\right|\left|Z_{0}\right|\right)^{2}}\right)
$$

For $\left|Z_{0}\right|=\left|a_{0}\right|$, the annulus becomes a disc with

$$
\left|Z_{1}\right|_{\min }=0
$$

and

$$
W_{1}=\frac{2\left|a_{0}\right|^{2}}{1+\left|a_{0}\right|^{2}}
$$

As $\left|Z_{1}\right|$ touches zero the phase of $Z_{1}$ becomes undefined with crucial consequences. We will study them now.

Our aim is to compare the rates of rotation of $\psi_{0}$ and $\psi_{1}$ for the following domains of $\left|Z_{0}\right|=e^{-k r}$,

$$
\begin{aligned}
& \left|Z_{0}\right|>\left|a_{0}\right|, \\
& \left|Z_{0}\right|=\left|a_{0}\right|
\end{aligned}
$$


and

$$
\left|Z_{0}\right|<\left|a_{0}\right|
$$

For $\left|Z_{p}\right|=1\left(>\left|a_{p}\right|\right)$ we have already seen ((A.16) to (A.21)) that on the average $\psi_{p+1}$ turns twice as fast as $\psi_{p}$. In particular, $\psi_{1}$ turns twice as fast as $\psi_{0}$. This result will be seen to hold more genrally for $\left|Z_{0}\right|>\left|a_{0}\right|$. But discontinuities appear as $\left|Z_{0}\right|$ comes down to $\left|a_{0}\right|$ and crosses over. We will demonstrate this now.

Let us examine (A.42) for $p=0$ and in particular the term $\ln f_{1}$. For $\left|Z_{0}\right|>\left|a_{0}\right|$ the real part of $\left(\left|a_{0}\right|+\left|Z_{0}\right| e^{-i \chi_{0}}\right)$ can vanish and change sign. This feature is absent in $f_{2}$ since always $\left|a_{p}\right|\left|Z_{p}\right|<1$. So the term $\ln f_{1}$ has to be treated carefully to keep track of additive contributions as $\psi_{0}$ rotates ( $t$ increases). It is convenient to proceed as follows. For

$$
\begin{gathered}
\left|Z_{0}\right|>\left|a_{0}\right| \\
f_{1}=e^{-i 2 \chi_{0}}\left(\frac{1+\frac{\left|a_{0}\right|}{\left|Z_{0}\right|} e^{i \chi_{0}}}{1+\frac{\left|a_{0}\right|}{\left|Z_{0}\right|} e^{-i \chi_{0}}}\right)
\end{gathered}
$$

For

$$
\begin{gathered}
\left|Z_{0}\right|=\left|a_{0}\right| \\
f_{1}=e^{-i \chi_{0}}
\end{gathered}
$$

For

$$
\begin{gathered}
\left|Z_{0}\right|<\left|a_{0}\right| \\
f_{1}=\left(\frac{1+\frac{\left|Z_{0}\right|}{\left|a_{0}\right|} e^{-i \chi_{0}}}{1+\frac{\left|Z_{0}\right|}{\left|a_{0}\right|} e^{i \chi_{0}}}\right)
\end{gathered}
$$

Hence for the three domains, respectively,

$$
\begin{aligned}
\psi_{1} & =2 \psi_{0}+\Lambda_{(+)} \\
\psi_{1} & =\frac{3}{2} \psi_{0}+\Lambda_{(0)} \\
\psi_{1} & =\psi_{0}+\Lambda_{(-)}
\end{aligned}
$$

where one can now safely consider that

$$
\Lambda_{\delta}\left(\psi_{0}+2 \pi\right)=\Lambda_{\delta}\left(\psi_{0}\right), \quad(\delta=+, 0,-)
$$


Hence for $\psi_{0} \rightarrow \psi_{0}+2 n \pi$ there are no cumulative, additive contributions from $\Lambda_{\delta}$ giving a supplementary term proportional to $n$ (as does the first term proportional to $\left.\psi_{0}\right)$. The discontinuity involved for $\psi_{1}$ at $\left|Z_{0}\right|=\left|a_{0}\right|$ is now explicit.

In view of the crucial role of this result we present an alternative approach, closely following the argument for the circle map ((A.18) to (A.21)) but generalizing it for all $\left|Z_{0}\right|>\left|a_{0}\right|$. (This also generalizes the arguments for chaoticity on the circumference for the interior of the disc.)

It is sufficient to consider one single term $\left(f_{1}\right)$ as follows. Define

$$
e^{-i \beta}=\frac{\left|a_{0}\right|+\left|Z_{0}\right| e^{-i \chi_{0}}}{\left|a_{0}\right|+\left|Z_{0}\right| e^{i \chi_{0}}}
$$

when

$$
\frac{d \beta}{d \psi_{0}}=\frac{2\left|Z_{0}\right|\left(\left|Z_{0}\right|+\left|a_{0}\right| \cos \chi_{0}\right)}{\left(\left|a_{0}\right|^{2}+\left|Z_{0}\right|^{2}+2\left|a_{0}\right|\left|Z_{0}\right| \cos \chi_{0}\right)}
$$

Hence for $\left|Z_{0}\right|>\left|a_{0}\right|$ one has strict monotonicity with

$$
\frac{d \beta}{d \psi_{0}}>0
$$

but not for $\left|Z_{0}\right|<\left|a_{0}\right|$. For the exceptional value $\left|Z_{0}\right|=\left|a_{0}\right|$ one has simply

$$
e^{-i \beta}=e^{-i \chi_{0}}
$$

For $\left|Z_{0}\right|>\left|a_{0}\right|$ along with strict monotonicity one has

$$
\begin{aligned}
& e^{-i \beta}=-1 \text { for } \cos \chi_{0}=-\frac{\left|a_{0}\right|}{\left|Z_{0}\right|}, \sin \chi_{0}= \pm \sqrt{1-\frac{\left|a_{0}\right|^{2}}{\left|Z_{0}\right|^{2}}} \\
& e^{-i \beta}=1 \text { for } \cos \chi_{0}=0, \pi, 2 \pi
\end{aligned}
$$

Hence, as for the circle map, "on the average" $\beta$ turns twice as fast as $\psi_{0}$. This corresponds to the factor 2 in the first equation of (A.52), namely,

$$
\psi_{1}=2 \psi_{0}+\Lambda_{(+)}
$$

Now suppose we consider the Poincaré sections for

$$
t_{n}=t+n T \quad(n=0,1,2, \ldots)
$$


with some suitably chosen period $T$. The "rotation number" for $\psi_{0}(=k t)$ is defined in terms of

$$
\psi_{0}^{(n)}=k t_{n}
$$

as

$$
\Omega_{0}=\left(n^{-1}\left(\psi_{0}^{(n)}-\psi_{0}\right)\right)_{n \rightarrow \infty}=k T
$$

(We reserve the term "winding number" for the magnetic charge $q$ of the gauge fields.) Here, of course, it would have been natural to set

$$
T=2 \pi k^{-1}
$$

or, rescaling, obtain an integer (say, unit) value for $\Omega_{0}$. But we keep $T$ unspecified here since for the gauge fields there will be the simultaneous presence of different periodic building blocks each with it's own period, which may even be mutually incommensurable. (See Sec.3.) Now, similarly defining the rotation number $\Omega_{1}$ for $\psi_{1}$, one obtains from (A.52) and(A.53)

$$
\begin{array}{llll}
\Omega_{1} & =2 \Omega_{0} & \text { for } & e^{-k r}>\left|a_{0}\right| \\
\Omega_{1}=\frac{3}{2} \Omega_{0} & \text { for } & e^{-k r}=\left|a_{0}\right| \\
\Omega_{1}=\Omega_{0} & \text { for } & e^{-k r}<\left|a_{0}\right|
\end{array}
$$

Hence there is a step discontinuity at $r=-k^{-1} \ln \left|a_{0}\right|$. Let us further examine how this implies sensitive dependence on small differences in the value of a parameter. Let $\psi_{1}^{( \pm)}$and $\hat{\psi}_{1}$ denote the values of $\psi_{1}$ respectively for

$$
\left|Z_{0}\right|=\left|a_{0}\right|(1 \pm \varepsilon) \text { and }\left|a_{0}\right|
$$

Here $\varepsilon \ll 1$. From (A.42) with $p=0$, upto $\mathrm{O}(\epsilon)$, one obtains

$$
\psi_{1}^{( \pm)}=\hat{\psi}_{1} \pm \varepsilon\left(\frac{1}{2} \tan \frac{\chi_{0}}{2}-\frac{\left|a_{0}\right|^{2} \sin \chi_{0}}{1+\left|a_{0}\right|^{4}+2\left|a_{0}\right|^{2} \sin \chi_{0}}\right)
$$

But such a development is valid if not only $\varepsilon \ll 1$ but also ctan $\frac{\chi_{0}}{2} \ll 1$. Even if one starts with a suitably small value of the latter, the constraint will be violated as $\chi_{0}$ approaches the values $(2 N+1) \pi$. Near such values one may proceed as follows. The contribution to $-i 2\left(\psi_{1}^{(+)}-\psi_{1}^{(-)}\right)$from the term $\ln f_{1}$ 
in $(\mathrm{A} .42)$ is

$$
\ln \left(\left(\frac{1+e^{i \chi_{0}}+\varepsilon}{1+e^{-i \chi_{0}}+\varepsilon}\right)\left(\frac{1+e^{-i \chi_{0}}-\varepsilon}{1+e^{i \chi_{0}}-\varepsilon}\right)\right)=\ln \left(\frac{\cos \left(\chi_{0} / 2\right)-i(\varepsilon / 2) \sin \left(\chi_{0} / 2\right)}{\cos \left(\chi_{0} / 2\right)+i(\varepsilon / 2) \sin \left(\chi_{0} / 2\right)}\right)
$$

For $\chi_{0}=(2 N+1) \pi$ this is no longer proportional to $\varepsilon$ but becomes $\ln (-1)$. The additive contibution at each turn gives a difference $\left(\psi_{1}^{(+)}-\psi_{1}^{(-)}\right)$consistent with (A.59).

So far we have analysed the effect of the passage $Z_{0} \rightarrow Z_{1}$. For the next step, write

$$
Z_{2}=\left(\frac{a_{1}+Z_{1}}{\bar{a}_{1}+Z_{1}^{-1}}\right)=Z_{0}^{-2}\left(\frac{a_{0}+Z_{0}}{\bar{a}_{0}+Z_{0}^{-1}}\right)\left(\frac{\mu_{+}+Z_{0}}{\bar{\mu}_{+}+Z_{0}^{-1}}\right)\left(\frac{\mu_{-}+Z_{0}}{\bar{\mu}_{-}+Z_{0}^{-1}}\right)
$$

where (compare (A.11) and the discussion that follows)

$$
\mu_{ \pm}=\sqrt{a}_{1}\left(\lambda_{0} \pm \sqrt{\lambda_{0}^{2}-1}\right)
$$

with

$$
\lambda_{0}=\frac{a_{0}+\bar{a}_{0} a_{1}}{2 \sqrt{a}_{1}}
$$

One sees that for studying $\Omega_{2}$ (the rotation number associated to the phase $\psi_{2}$ of $Z_{2}$ ) one should extend the previous considerations to three spherical shells

$$
\left|Z_{0}\right|=\left|a_{0}\right|, \quad\left|\mu_{+}\right|, \quad\left|\mu_{-}\right|
$$

where

$$
\left|\mu_{+}\right|\left|\mu_{-}\right|=\left|a_{1}\right|
$$

One may get special features corresponding to a possible double zero when $\left|\mu_{+}\right|$and $\left|\mu_{-}\right|$coincide to $\sqrt{\left|a_{1}\right|}$. The number of possibilities continue to increase with each iteration. Generically the process is systematic, but roots can coincide leading to special features. The cumulative effects of the jumps give more and more elaborate staircase like patterns (Sec.2).

Considering $\left(\left|Z_{p}\right|, \psi_{p}\right)$ as independent variables one may carry out a similar analysis for the step $Z_{p} \rightarrow Z_{p+1}$ (for $p>0$ ). One then obtains formally, like (A.52) with the $\Lambda$ 's satisfying the criterion (A.53),

$$
\psi_{p+1}=2 \psi_{p}+\Lambda_{(+)}^{(p)}
$$




$$
\begin{aligned}
\psi_{p+1} & =\frac{3}{2} \psi_{p}+\Lambda_{(0)}^{(p)} \\
\psi_{p+1} & =\psi_{p}+\Lambda_{(-)}^{(p)}
\end{aligned}
$$

respectively for

$$
\left|Z_{p}\right|>\left|a_{p}\right|, \quad\left|Z_{p}\right|=\left|a_{p}\right|, \quad\left|Z_{p}\right|<\left|a_{p}\right| .
$$

But it should be clearly noted that for $p \geq 1$ all $\left|Z_{p}\right|$ 's are time dependent even for the choice (2) for $Z_{0}$. For the choice (3) even $Z_{0}$ is time dependent. The formal steps of the iterations and their consequences in the context of the mapping do not depend on the parametrization of $Z_{0}$ (provided it satifies $\left.Z_{0}<1\right)$. But when the time evolution is studied in the context of the gauge fields much depends evidently on the initial choice. 


\section{References}

[1] A. Chakrabarti, Periodic generalizations of static, selfdual $S U(2)$ gauge fields, Phys. Rev. D35, 696 (1987).

[2] A. Chakrabarti, Spinors in periodic selfdual gauge field backgrounds, Jour. Math. Phys. 29, 481 (1988).

[3] A. Chakrabarti, Propagators in periodic gauge field backgrounds, Phys. Lett. B210, 181 (1988).

[4] A. Chakrabarti, Quasiperiodic instantons, Phys. Rev. D38, 329 (1988).

[5] A. Chakrabarti and C. Roiesnel, Periodic and quasiperiodic $S U(N)$ instantons, Phys. Rev. D39, 2381 (1989).

[6] A. Chakrabarti, Antiperiodic spinor zero modes for periodic $S U(2)$ instanton backgrounds, Phys. Lett. B227, 137 (1989).

[7] A. Chakrabarti, Linear fluctuations of periodic and quasiperiodic instantons, Phys. Rev. D40, 2684 (1989).

[8] A. Chakrabarti, Quasiperiodic gauge fields: Poincaré sections, annular maps and irrational winding numbers (3 pages, unpublished talk presented at WIGSYM, Oxford) (1993).

[9] R. L. Devaney, An introduction to chaotic dynamical systems, Bejamin/ Cummings Pub; (1986).

[10] P. Bak, T. Bohr and M. H. Jensen, Circle maps, mode-locking and chaos ( Directions in chaos (vol 2), Ed. Ho Bai-lin, World Scientific, 1988).

[11] T. Geisel, Quasiperiodicity versus mixing instability in a kicked quantum system, Phys. Rev. A41, 2989 (1990).

[12] J. P. Eckmann and D. Ruelle, Ergodic theory of chaos and srange attractors, Rev. Mod. Phys. 57, 617 (1985).

[13] E. Witten, Some exact multipseudoparticle solutions of classical YangMills thoery, Phys. Rev. Lett. 38, 121 (1977). 
[14] R. Jackiw, C. Nohl and C. Rebbi, Conformal properties of pseudoparticle configurations, Phys. Rev. D15, 1642 (1977).

[15] L. S. Brown and D. B. Creamer, Vacuum polarization about instantons, Phys. Rev. D18, 3695 (1978).

[16] C. Roiesnel and A. Chakrabarti, Geometry of linear pairs for selfdual gauge fields, Jour. Math. Phys. 31, 1643 (1990).

[17] A. Chakrabarti, Costruction of hyperbolic monopoles, Jour. Math. Phys. 27, 340 (1986).

[18] A. Chakrabarti, Classical solutions of Yang-Mills fields (selected topics), Fortschr. Phys. 35, 1 (1987).

[19] T. S. Biró, S. G. Matinyan and B. Müller, Chaos and guage field theory, World Scientific (1994).

[20] C. Mukku, M. S. Sriram, J. Segar, B. A. Bambah and S. Lakshmibala, Order-chaos transition in field theories with topological terms: a dynamical system approach, (hep-th/9610071), Oct 1996.

[21] Shau-Jin Chang, Classical Yang-Mills solutions and iterative maps, Phys. Rev. D29, 259 (1984).

[22] S. G. Matinyan, E. B. Prokhorenko and G. K. Savvidy, Non-integrability of time dependent spherically symmetric Yang-Mills equations, Nucl. Phys. B298, 414 (1988).

[23] B. K. Harrington and H. K. Shepard, Periodic Euclidean solutions and the finite temperature Yang-Mills gas, Phys. Rev. D17, 2122 (1978).

[24] B. K. Harrington and H. K. Shepard, Thermodynamics of Yang-Mills gas, Phys. Rev. D18, 2990 (1978).

[25] D. J. Gross, R. D. Pisarski and L. G. Yaffe, QCD and instantons at finite temperature, Rev. Mod. Phys. 53, 43 (1981). 